\title{
BET bromodomain inhibitors and agonists of the beta-2 adrenergic receptor identified in screens for compounds that inhibit DUX4 expression in FSHD muscle cells
}

Amy E. Campbell', Jonathan Oliva², Matthew P. Yates ${ }^{2}$, Jun Wen Zhong ${ }^{1}$, Sean C. Shadle ${ }^{1,3}$, Lauren Snider ${ }^{1}$, Nikita Singh ${ }^{2}$, Shannon Tai ${ }^{2}$, Yosuke Hiramuki ${ }^{1}$, Rabi Tawil ${ }^{4}$, Silvère M. van der Maarel ${ }^{5}$, Stephen J. Tapscott ${ }^{1,6^{*}}$ (i) and Francis M. Sverdrup ${ }^{2 *}$

\begin{abstract}
Background: Facioscapulohumeral dystrophy (FSHD) is a progressive muscle disease caused by mutations that lead to epigenetic derepression and inappropriate transcription of the double homeobox 4 (DUX4) gene in skeletal muscle. Drugs that enhance the repression of DUX4 and prevent its expression in skeletal muscle cells therefore represent candidate therapies for FSHD.

Methods: We screened an aggregated chemical library enriched for compounds with epigenetic activities and the Pharmakon 1600 library composed of compounds that have reached clinical testing to identify molecules that decrease DUX4 expression as monitored by the levels of DUX4 target genes in FSHD patient-derived skeletal muscle cell cultures.

Results: Our screens identified several classes of molecules that include inhibitors of the bromodomain and extra-terminal (BET) family of proteins and agonists of the beta-2 adrenergic receptor. Further studies showed that compounds from these two classes suppress the expression of DUX4 messenger RNA (mRNA) by blocking the activity of bromodomain-containing protein 4 (BRD4) or by increasing cyclic adenosine monophosphate (cAMP) levels, respectively.

Conclusions: These data uncover pathways involved in the regulation of DUX4 expression in somatic cells, provide potential candidate classes of compounds for FSHD therapeutic development, and create an important opportunity for mechanistic studies that may uncover additional therapeutic targets.
\end{abstract}

Keywords: Facioscapulohumeral muscular dystrophy, FSHD, DUX4, Bromodomain, Adrenergic, High-throughput screening

\footnotetext{
* Correspondence: stapscot@fredhutch.org; fran.sverdrup@health.slu.edu ${ }^{1}$ Human Biology Division, Fred Hutchinson Cancer Research Center, Seattle, WA 98109, USA

${ }^{2}$ Edward A. Doisy Department of Biochemistry and Molecular Biology, Saint

Louis University, Saint Louis, MO 63104, USA

Full list of author information is available at the end of the article
} 


\section{Background}

Facioscapulohumeral dystrophy (FSHD) is a prevalent muscular dystrophy affecting over 800,000 individuals worldwide. The disease typically presents in young adults as facial and upper extremity weakness, and progresses to involve nearly all skeletal muscle groups [1]. FSHD is caused by the mis-expression of the double homeobox 4 (DUX4) transcription factor in skeletal muscle. DUX4 is encoded by a retrogene located in each unit of the D4Z4 macrosatellite repeat array in the subtelomeric region of chromosomes $4 \mathrm{q}$ and $10 \mathrm{q}$, and is normally expressed in the pre-implantation embryo and in germline tissues where it activates early developmental and stem cell genes [1-4]. In most somatic tissues, including skeletal muscle, the D4Z4 arrays and DUX4 are epigenetically silenced through multiple mechanisms that suppress repetitive elements in the genome [5-9].

FSHD results from a contraction at $4 \mathrm{q} 35$ resulting in too few $\mathrm{D} 4 \mathrm{Z} 4$ repeats for efficient repeat-mediated epigenetic repression (FSHD type 1, FSHD1) or from the presence of mutations in trans-acting chromatin factors necessary for epigenetic repression of the D4Z4 array (FSHD type 2, FSHD2) [10-12]. Inefficient D4Z4 repression, when combined with a permissive chromosome 4qA haplotype that provides a polyadenylation site for the DUX4 messenger RNA (mRNA), results in the ectopic expression of DUX4 protein in muscle cells $[1,5,10]$. DUX4 mis-expression in skeletal muscle induces early embryo, stem cell, and germline genes; activates repetitive elements; suppresses innate immune response and nonsense-mediated RNA decay pathways; inhibits myogenesis; and causes cell death through mechanisms that include the accumulation of aberrant and double-stranded RNAs [13-22].

Because of its causative role in FSHD, suppressing DUX4 expression is a primary therapeutic approach for halting disease progression. However, the mechanisms responsible for DUX4 expression are poorly understood and limited drug targets have been identified. Consequently, there is currently no treatment available for FSHD and few clinical trials of promising therapies are ongoing.

Here, we screened an aggregated chemical library enriched for compounds with epigenetic activities and the Pharmakon 1600 library composed of compounds that have reached clinical testing to identify molecules that decrease DUX4 expression as monitored by the levels of DUX4 target genes in FSHD patient-derived muscle cells. Our screens identified bromodomain and extra-terminal (BET) bromodomain inhibitors and beta-2 adrenergic receptor agonists as classes of compounds that suppress DUX4 expression. These findings illuminate pathways that regulate $D U X 4$ expression in somatic cells and provide initial candidate molecules for FSHD therapeutic development.

\section{Methods \\ Compounds}

The Pharmakon 1600 drug library was obtained from MicroSource Discovery Systems, Inc. (Gaylordsville, CT, USA). The collection of epigenetic modulator compounds was composed of the Epigenetics Screening Library from Cayman Chemical (Ann Arbor, MI, USA), the Epigenetics Compound Library from Selleck Chemicals LLC (Houston, TX, USA), and novel epigenetic probes acquired from the Structural Genomics Consortium (www.thesgc.org). Screening compounds were delivered in microplates as $10 \mathrm{mM}$ stocks dissolved in dimethyl sulfoxide (DMSO) and kept at $-80{ }^{\circ} \mathrm{C}$ until use. Individual compounds used in follow-up testing were purchased from Sigma-Aldrich (St. Louis, MO, USA), Tocris Bioscience (Bristol, UK), or Selleck Chemicals, dissolved in DMSO at a $10 \mathrm{mM}$ stock concentration and stored at $-80{ }^{\circ} \mathrm{C}$.

\section{Cell culture}

Primary human myoblast cell lines were obtained from the Fields Center at the University of Rochester (https://www.urmc.rochester.edu/neurology/fields-center.aspx) and immortalized by retroviral transduction of cyclin-dependent kinase 4 (CDK4) and human telomerase reverse transcriptase (hTERT) [23]. Immortalized myoblasts were grown in Ham's F-10 Nutrient Mix (Gibco, Waltham, MA, USA) supplemented with 20\% HyClone Fetal Bovine Serum (GE Healthcare Life Sciences, Pittsburgh, PA, USA), $100 \mathrm{U} / 100 \mu \mathrm{g}$ penicillin/streptomycin (Gibco), $10 \mathrm{ng} / \mathrm{ml}$ recombinant human fibroblast growth factor (Promega Corporation, Madison, WI, USA), and $1 \mu \mathrm{M}$ dexamethasone (SigmaAldrich). Differentiation of myoblasts into myotubes was achieved by switching the fully confluent myoblast monolayer into Dulbecco's Modified Eagle Medium (DMEM) (Gibco) containing 1\% horse serum (Gibco), $100 \mathrm{U} / 100 \mu \mathrm{g}$ penicillin/streptomycin, $10 \mu \mathrm{g} / \mathrm{ml}$ insulin (Sigma-Aldrich), and $10 \mu \mathrm{g} / \mathrm{ml}$ transferrin (Sigma-Aldrich) (HS/IT media) or DMEM/Nutrient Mixture F-12 (1:1, Gibco) supplemented with $2 \%$ KnockOut Serum Replacement (Gibco), $100 \mathrm{U} / 100 \mu \mathrm{g}$ penicillin/streptomycin, $10 \mu \mathrm{g} / \mathrm{ml}$ insulin, and $10 \mu \mathrm{g} / \mathrm{ml}$ transferrin (KSR media) for 2-6 days. The details of each cell line used in this study are provided in Additional file 1: Table S1.

\section{Epigenetic modifier library screen}

Transient DNA transfections of 54-2 FSHD1 myoblasts were performed using TransIT-2020 (Mirus Bio LLC, Madison, WI, USA) according to the manufacturer's specifications. Briefly, $15 \times 10^{6}$ cells were suspended in $25 \mathrm{ml}$ 
growth media on the day of transfection. Cells were cotransfected with $16.2 \mu \mathrm{g}$ of a pGL3-basic reporter vector carrying the intact or mutated zinc finger and SCAN domain-containing 4 (ZSCAN4) promoter fragment upstream of the firefly luciferase gene [13] and $1.8 \mu \mathrm{g}$ of a pRL-CMV-Renilla luciferase control reporter vector. DNAs were diluted in $1.8 \mathrm{ml}$ Opti-MEM Reduced Serum Medium (Thermo Fisher Scientific, Waltham, MA, USA) with $45 \mu \mathrm{l}$ TransIT-2020 transfection reagent. Suspended cells were combined with the transfection mixture and plated in a T175 flask. The next day, cells were trypsinized and re-plated at $5 \times 10^{4}$ cells/well in 96-well plates. Growth media was removed and replaced with HS/IT differentiation media the following day. Compounds were added the next day and cells were allowed to differentiate for an additional $96 \mathrm{~h}$. Luminescence was quantified using the Dual-Luciferase Reporter Assay System (Promega) and a SpectraMax L microplate luminometer (Molecular Devices LLC, Sunnyvale, CA, USA) following the manufacturers' directions.

\section{Pharmakon 1600 library screen}

Compounds were diluted 1:2000 from DMSO stocks into KSR differentiation media so that DMSO was limited to $0.05 \%$, and the initial screening concentration was $5 \mu \mathrm{M}$ with one well per compound. Media containing compounds was added to confluent monolayers of MB200 FSHD2 myoblasts in 96-well plates. Forty-two hours later, each well was visually inspected and documented before cells were harvested for mRNA expression analysis. Each 96-well screening plate contained 8 positive (I-BET762, $1 \mu \mathrm{M}$ ) and 8 negative (DMSO) controls such that every row included one of each type of control. The sequential addition of cell lysis reagents, which proceeded by row, resulted in slight variations in the maximal and minimal PCR signals from row to row. Consequently, a marginal Z-prime of 0.217 was calculated from the positive and negative controls for the entire screen (20 plates) with a coefficient of variation of 0.23 . To compensate for this variability, data in each row were normalized to the negative control in that row and hits selected as compounds that resulted in $>65 \%$ target mRNA inhibition relative to the negative control. A plot of the primary screening data after row by row normalization is provided in Additional file 2: Figure S1.

\section{DUX4 activity assay}

Briefly, $6 \times 10^{5} 54-6$ control (non-FSHD) myoblasts in 1 well of a 6-well plate were co-transfected with $75 \mathrm{ng}$ of the DUX4 expression vector pCS2-DUX4 [13] and $2.925 \mu \mathrm{g}$ of pGL3-promoter vector (Promega) using Lipofectamine 3000 (Thermo Fisher Scientific) following the manufacturer's instructions. Three hours after transfection, cells were trypsinized and distributed to 84 wells of a 96-well plate. Two hours later (a time at which there is a low but detectable level of DUX4 target gene expression), 12 wells were harvested to represent the "baseline" gene expression state while compounds were added to the remaining wells. Nineteen hours later, the remaining wells were harvested to represent the "endpoint" gene expression state. DUX4 activity was determined by comparing DUX4 target gene mRNA levels at the $24 \mathrm{~h}$ "endpoint" to the levels at the $5 \mathrm{~h}$ "baseline."

\section{mRNA expression analyses}

For screening of the Pharmakon 1600 library and for the DUX4 activity assay, cell lysates were prepared using Cells-to-Ct Bulk Lysis Reagents (Invitrogen, Waltham, MA, USA). Quantitative polymerase chain reaction (PCR) was carried out on a QuantStudio 5 (Applied Biosystems, Waltham, MA, USA) using TaqMan Gene Expression Assays (Applied Biosystems) and TaqMan Fast Virus 1-Step Master Mix (Invitrogen). The relative expression level of DUX4 target gene methyl-CpG binding domain protein 3 like 2 (MBD3L2) was normalized to that of the reference gene ribosomal protein L30 (RPL30), which was included in multiplex (two gene) PCR reactions. The pair performed well in terms of amplification efficiency. For other gene expression analyses, total RNA was extracted from whole cells using the RNeasy Mini Kit (Qiagen, Hilden, Germany) according to the manufacturer's instructions. Isolated RNA was treated with DNase I (Thermo Fisher Scientific), heat inactivated, and reverse transcribed into cDNA using Superscript III (Thermo Fisher Scientific) and oligo(dT) primers (Invitrogen) following the manufacturer's protocol. Quantitative PCR was carried out on a QuantStudio 7 Flex (Applied Biosystems) using primers specific for each mRNA and iTaq SYBR Green Supermix (Bio-Rad Laboratories, Inc., Hercules, CA, USA), or on a QuantStudio 5 using TaqMan Gene Expression Assays and TaqMan Gene Expression Master Mix (Applied Biosystems). The relative expression levels of target genes were normalized to that of the reference genes ribosomal protein L27 (RPL27), ribosomal protein L13a (RPL13A), or RPL30 by using the delta-delta-Ct method [24] after confirming equivalent amplification efficiencies of reference and target molecules.

\section{DNA methylation analysis}

DNA methylation analyses were conducted by EpigenDx, Inc. (Hopkinton, MA, USA). Genomic DNA specimens were isolated using the QIAamp DNA Mini Kit (Qiagen) and shipped on dry ice from Saint Louis University to EpigenDx. For each sample, 500 ng of extracted genomic DNA was bisulfite treated using the EZ DNA Methylation Kit (Zymo Research, Irvine, CA, USA). Bisulfite-treated DNA was purified according to the manufacturer's protocol and eluted in a final volume of $46 \mu \mathrm{l}$. PCR was 
performed using $1 \mu \mathrm{l}$ of bisulfite-treated DNA and $0.2 \mu \mathrm{M}$ of each primer for EpigenDx methylation assays ADS3747 and ADS1454. One primer was biotin-labeled and highperformance liquid chromatography-purified in order to capture the final PCR product using sepharose beads. For pyrosequencing, PCR products were bound to Streptavidin Sepharose High Performance (GE Healthcare Life Sciences), after which the immobilized PCR products were purified, washed, denatured with a $0.2 \mu \mathrm{M} \mathrm{NaOH}$ solution, and rewashed using the Pyrosequencing Vacuum Prep Tool (Qiagen) per the manufacturer's protocol. Next, $0.5 \mu \mathrm{M}$ of sequencing primer was annealed to the purified single-stranded PCR products. Ten microliters of the PCR products were sequenced by pyrosequencing on the PSQ96 HS System (Qiagen) following the manufacturer's instructions. The methylation status of each $\mathrm{CpG}$ site was determined individually as an artificial $\mathrm{C} / \mathrm{T}$ single nucleotide polymorphism using the QCpG software (Qiagen). The methylation level at each CpG site was calculated as the percentage of the methylated alleles divided by the sum of all methylated and unmethylated alleles. The mean methylation level was calculated using methylation levels of all measured $\mathrm{CpG}$ sites within the targeted region of each gene. Each experiment included non-CpG cytosines as internal controls to detect incomplete bisulfite conversion of the input DNA. In addition, a series of unmethylated and methylated DNA were included as controls in each PCR. Furthermore, PCR bias testing was performed by mixing unmethylated control DNA with in vitro methylated DNA at different ratios $(0 \%, 5 \%, 10 \%, 25 \%, 50 \%$, $75 \%, 100 \%$ ), followed by bisulfite modification, PCR, and pyrosequencing analysis.

\section{Small interfering RNA transfections}

Duplex small interfering RNAs (siRNAs) were obtained from Qiagen (FlexiTube) or Thermo Fisher Scientific (Silencer Select). Transfections of siRNAs into myoblasts were carried out using Lipofectamine RNAiMAX (Invitrogen) or Lipofectamine 2000 (Invitrogen) according to the manufacturer's instructions. For bromodomain-containing protein 2 (BRD2), bromodomain-containing protein 3 (BRD3), and bromodomain-containing protein 4 (BRD4) siRNAs, $1.5 \times 10^{5}$ cells suspended in $1 \mathrm{ml}$ culture media were mixed with $2 \mu \mathrm{l}$ Lipofectamine 2000 and $12.5 \mathrm{pmol}$ of either gene-specific siRNAs or a scrambled nonsilencing control siRNA diluted in $100 \mu \mathrm{l}$ Opti-MEM Reduced Serum Medium and plated in 1 well of a 12-well plate. Cells were harvested for RNA analysis 72-96 h later. For adrenoceptor beta 2 (ADRB2) siRNA, a double transfection protocol was followed to ensure efficient depletion of pre-existing proteins. Briefly, cells were seeded at $\sim 30 \%$ confluence in 6-well plates and transfected $\sim 20 \mathrm{~h}$ later with $6 \mu$ Lipofectamine RNAiMAX and 25 pmol of either gene-specific siRNAs or a scrambled non-silencing control siRNA diluted in $125 \mu \mathrm{l}$ Opti-MEM Reduced Serum Medium. Forty-eight hours following this, cells were transfected a second time and harvested for RNA analysis $48-72 \mathrm{~h}$ later.

\section{Western blotting}

Reduced and boiled samples were run on NuPage 4-12\% precast polyacrylamide gels (Invitrogen) and transferred to polyvinylidene difluoride membrane (Invitrogen). After blocking in 5\% milk in PBST, membranes were incubated with appropriate primary antibodies in block solution overnight at $4{ }^{\circ} \mathrm{C}$. Membranes were then incubated with horseradish peroxidase-conjugated secondary antibodies in block solution for $1 \mathrm{~h}$ at room temperature, and chemiluminscent substrate (Thermo Fisher Scientific) was used for detection.

\section{Antibodies}

The following antibodies were used: $\alpha$-Tubulin (DM1A), Sigma-Aldrich T9026; $\beta 2-A R$ (R11E1), Santa Cruz Biotechnology (Dallas, TX, USA) sc-81577, lot\#G1117; BRD2, Bethyl Laboratories (Montgomery, TX, USA) A302-582A; BRD3, Bethyl A302-368A; BRD4, Bethyl A301-985A50; GAPDH, Thermo Fisher Scientific TAB1001; Histone H3, Abcam (Cambridge, UK) ab1791; and MBD3L2, Abcam ab107999, lot\#GR126890-1; rabbit monoclonal antibody against DUX4 (E14-3) was produced in collaboration with Epitomics (Burlingame, CA, USA) and is described elsewhere [25].

\section{TaqMan gene expression assay ID numbers}

BRD2, Hs01121986_g1; BRD3, Hs00201284_m1; BRD4, Hs04188087_m1; BRDT, Hs00976114_m1; MBD3L2, Hs00544743_m1; MYF5, Hs00929416_g1; MYH2, Hs00430042_m1; MYOD1, Hs00159528_m1; MYOG, Hs01072232_m1; RPL13A, Hs04194366_g1; RPL30, Hs00265497_m1; SMCHD1, Hs00826906_m1; TRIM43, Hs00299174_m1; ZSCAN4, Hs00537549_m1; DUX4, primers GCCGGCCCAGGTACCA and CAGCG AGCTCCCTTGCA with probe 6FAMCAGTGCGCACC CCGMGBNFQ.
Oligonucleotide sequences
Quantitative reverse transcription $P C R$ primers
ADRB2 F: GCCTGTGCTGATCTGGTCAT.
ADRB2 R: AATGGAAGTCCAAAACTCGCA.
CKM F: CACCCCAAGTTCGAGGAGAT.
CKM R: AGCGTTGGACACGTCAAATA.
DUX4 F: GGCCCGGTGAGAGACTCCACAC.
DUX4 R: CCAGGAGATGTAACTCTAATCCAGGTT
TGC.
DUX4 transgene F: TGACTGGATATGTTGTGTTT
TAC.
DUX4 transgene R: CAACCCCGGATCCTTAGTG. 
MBD3L2 F: GCGTTCACCTCTTTTCCAAG. MBD3L2 R: GCCATGTGGATTTCTCGTTT. MYOG F: GCCAGACTATCCCCTTCCTC. MYOG R: GAGGCCGCGTTATGATAAAA. RPL27 F: GCAAGAAGAAGATCGCCAAG. RPL27 R: TCCAAGGGGATATCCACAGA. RPL13A F: AACCTCCTCCTTTTCCAAGC. RPL13A R: GCAGTACCTGTTTAGCCACGA. TRIM43 F: ACCCATCACTGGACTGGTGT. TRIM43 R: CACATCCTCAAAGAGCCTGA. ZSCAN4 F: TGGAAATCAAGTGGCAAAAA. ZSCAN4 R: CTGCATGTGGACGTGGAC.

\section{siRNA target sequences}

ADRB2 \#1: GAGGGTAATAAACTTAGAATA (FlexiTube). ADRB2 \#2: CCAGGATAACCTCATCCGTAA (FlexiTube). BRD2: GGTCTACCGGATTATCACA (Silencer Select). BRD3: CGGCTGATGTTCTCGAATT (Silencer Select). BRD4 \#1: AGATTGAAATCGACTTTGA (Silencer Select). BRD4 \#2: TGAGCACAATCAAGTCTAA (Silencer Select). Negative control \#1: Silencer Select Negative Control \#1. Negative control \#2: AATTCTCCGAACGTGTCACGT (FlexiTube).

\section{Results}

High-throughput screening approach to target DUX4 expression

Detection of DUX4 mRNA in FSHD muscle cells for drug screening purposes is challenging due to low transcript levels that reflect the fact that DUX4 is expressed in only approximately 1 in 1000 FSHD myoblasts in culture [5], and that DUX4 mRNA is a target of nonsensemediated decay [15] with a short half-life. Additionally complicating quantitative detection of the rare DUX4 mRNA are long sense and antisense transcripts that extend through the D4Z4 arrays on chromosome $4 \mathrm{q}$ and the nearly identical D4Z4 arrays on chromosome 10q, as well as an alternative splice form of DUX4 (DUX4s) that produces a truncated protein without transcriptional activity $[5,13,21]$. And although DUX4 expression increases upon in vitro differentiation of proliferating FSHD myoblasts into multinucleated myotubes [26], the levels remain low and the dynamic variability is challenging for robust large-scale screening approaches.

Conversely, genes regulated by DUX4, most of which are undetectable in normal muscle but readily measurable in FSHD myoblasts and myotubes, provide abundant and stable RNAs that are sensitive and highly specific markers of DUX4 expression [13, 27]. Further, reporter constructs derived from promoters of DUX4-responsive genes provide an additional readout of DUX4 expression [13]. Thus, these target genes and derived reporters serve as excellent indicators of DUX4 mRNA and protein for use in high- throughput screens [28]. We carried out two such screens, differing primarily in the content of the compound libraries with minor distinctions in outcome measures, using FSHD muscle cells differentiated in culture to identify molecules that decrease DUX4 target gene induction.

\section{Screening identifies BET bromodomain inhibitors as blocking DUX4 expression}

In the first screen, we compiled several compound libraries that target epigenetic modifier proteins, representing about 100 modulators of known epigenetic "writers," "readers," and "erasers." The identities of all the compounds in our final collection are included in Additional file 3: Table S2. We employed 54-2 FSHD1 muscle cells transiently transfected with a luciferase reporter containing the promoter of the DUX4 target gene ZSCAN4 or a control reporter in which three of the four DUX4 binding sites present in the ZSCAN4 promoter were mutated (Additional file 4: Figure S2A) [13]. Luciferase activity was detected when using the ZSCAN4 reporters in 54-2 FSHD1 but not 54-6 control (non-FSHD) cells, and was dependent on intact DUX4 binding sites (Additional file 4: Figure S2B). The library compounds were screened at an initial concentration of $10 \mu \mathrm{M}$, or for some well-defined chemical probes at concentrations relevant for cell-based assays, for their ability to modulate the induction of the ZSCAN4 promoter in differentiating 54-2 FSHD1 myotubes. A co-transfected internal control plasmid that expressed Renilla luciferase was used to monitor general compound toxicities as well as non-specific transcriptional effects. Variability in the luminescence assay precluded achievement of acceptable Z-scores for single-well compound screening. Therefore, compounds were screened in replicates of eight and visual inspection was included as a qualitative criterion for determining toxicity. In order to prioritize compounds for follow-up study, we ranked the average of the 8 replicate values and selected those molecules that inhibited ZSCAN4 reporter expression > 50\% (after normalization to internal control Renilla luciferase activity), had $<25 \%$ effect independently on Renilla luciferase activity, and showed no obvious cytotoxicity (evident morphological change or loss of cell adhesion) upon visual examination.

This screening process identified four bromodomaininhibiting compounds (Table 1): CBP/p300 bromodomain inhibitor SGC-CBP30, pan-bromodomain inhibitor bromosporine, and BET bromodomain inhibitors (BETi) $(+)-J Q 1$ and PFI-1. Dilution curves revealed that $(+)-J Q 1$ and PFI-1 completely prevented activation of the ZSCAN4 promoter at high doses $(>1 \mu \mathrm{M})$ and resulted in half maximal effective concentrations $\left(\mathrm{EC}_{50} \mathrm{~s}\right)$ consistent with the reported cellular potencies for each BETi (Fig. 1a) [29, 30]. 
Table 1 Hits from the epigenetic modifier screen

\begin{tabular}{llll}
\hline Compound & $\begin{array}{l}\text { \% inhibition of } \\
\text { ZSCAN4 reporter }\end{array}$ & $\begin{array}{l}\text { \% inhibition of } \\
\text { Renilla luciferase }\end{array}$ & Mechanism \\
\hline (+)-JQ1 & $99\left(1 \mu \mathrm{M}^{\mathrm{a}}\right)$ & $27\left(1 \mu \mathrm{M}^{\mathrm{a}}\right)$ & $\begin{array}{l}\text { BET bromodomain } \\
\text { inhibitor }\end{array}$ \\
PFI-1 & $89\left(1 \mu \mathrm{M}^{\mathrm{a}}\right)$ & $0\left(1 \mu \mathrm{M}^{\mathrm{a}}\right)$ & $\begin{array}{l}\text { BET bromodomain } \\
\text { inhibitor }\end{array}$ \\
SGC-CBP30 & $75\left(1 \mu \mathrm{M}^{\mathrm{a}}\right)$ & $24\left(1 \mu \mathrm{M}^{\mathrm{a}}\right)$ & $\begin{array}{l}\text { CBP/p300 } \\
\text { bromodomain } \\
\text { inhibitor }\end{array}$ \\
& $92\left(2 \mu \mathrm{M}^{\mathrm{a}}\right)$ & $9\left(2 \mu \mathrm{M}^{\mathrm{a}}\right)$ & $\begin{array}{l}\text { Pan-bromodomain } \\
\text { inhibitor }\end{array}$ \\
& & &
\end{tabular}

${ }^{\mathrm{a} C o n c e n t r a t i o n ~ o f ~ c o m p o u n d ~ u s e d ~ i n ~ t h e ~ a s s a y ~}$

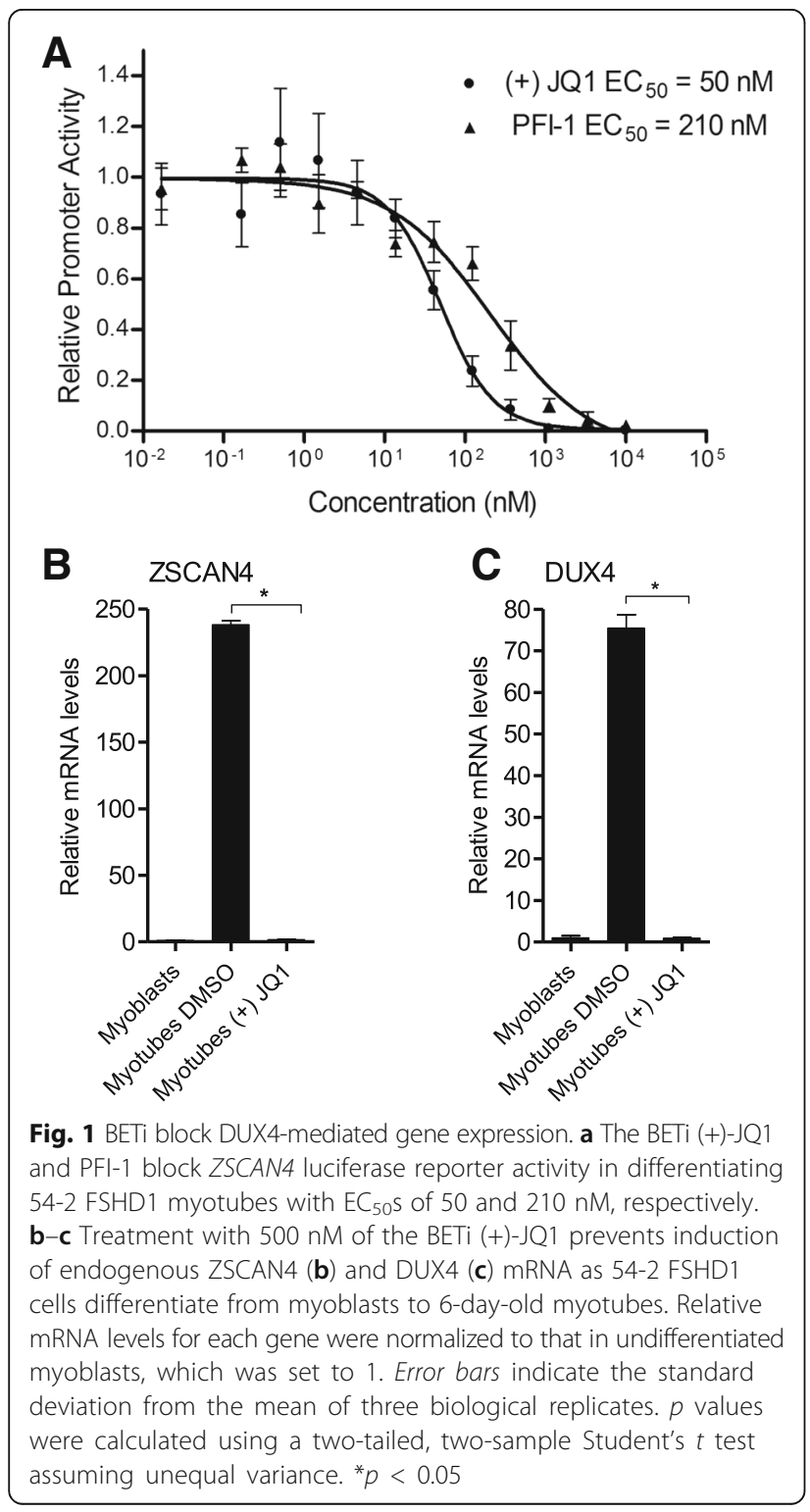

The luciferase readout in this screen was designed to reflect the expression of DUX4, which in turn modulates the ZSCAN4 promoter. To determine whether BETi as a class block induction of DUX4, we measured DUX4 and DUX4 target gene mRNA levels after compound treatment in 54-2 FSHD1 and MB200 FSHD2 proliferating myoblast and differentiating myotube cell cultures using quantitative reverse transcription PCR (RT-qPCR). The BETi probe compound (+)-JQ1, the clinical candidate I-BET151, the Phase II clinical molecule I-BET762, and the Phase III clinical compound RVX-208 prevented the activation of DUX4 and DUX4 target genes such as ZSCAN4 and tripartite motif containing 43 (TRIM43) under all conditions tested (Fig. 1b-c, Additional file 5: Figure S3). These results demonstrate that BETi block DUX4 expression in a manner independent of muscle differentiation in both FSHD1 and FSHD2 cells, suggesting that BETi act at a point in the transcriptional control of DUX4 common to both genetic defects.

To further validate that BETi function primarily by inhibiting the expression of DUX4 as opposed to affecting DUX4's ability to induce target genes, we ectopically expressed DUX4 in 54-6 control (non-FSHD) myoblasts, added BETi at concentrations up to 20-fold higher than needed to block DUX4 expression in FHSD myoblasts, and measured endogenous levels of the DUX4 target gene MBD3L2. BETi, even at high concentrations, did not block the ability of DUX4 to transactivate $M B D 3 L 2$ (Additional File 6: Figure S4). These results further support a mechanism by which BETi block DUX4 expression, not activity, and substantiate the use of DUX4 target genes to monitor BETi activity.

BET inhibition results in sustained suppression of DUX4 expression that is mediated by lysine deacetylation but not DNA methylation

We determined that treating FSHD1 and FSHD2 myoblasts with BETi resulted in decreased DUX4 target gene expression that manifested over the course of several days, with maximal inhibition apparent after $48-72 \mathrm{~h}$. To establish the time of drug exposure required to achieve the maximal response on DUX4 target gene expression, MB200 FSHD2 myoblasts were treated with $1 \mu \mathrm{M} \mathrm{I}$ BET762 for various amounts of time up to $72 \mathrm{~h}$ (Fig. 2a). For exposures shorter than $72 \mathrm{~h}$, drug-containing media was removed, the cells were rinsed before replacing fresh media without drug, and incubation continued until the 72-h time point. A 24-h pulse of I-BET762 resulted in sustained inhibition of the DUX4 targets ZSCAN4, TRIM43, and $M B D 3 L 2$, while an 8-h exposure was less effective. In contrast, mRNAs for the myoblast lineage markers myogenic differentiation 1 (MYOD1) and myogenic factor 5 (MYF5) and the epigenetic modifier structural 

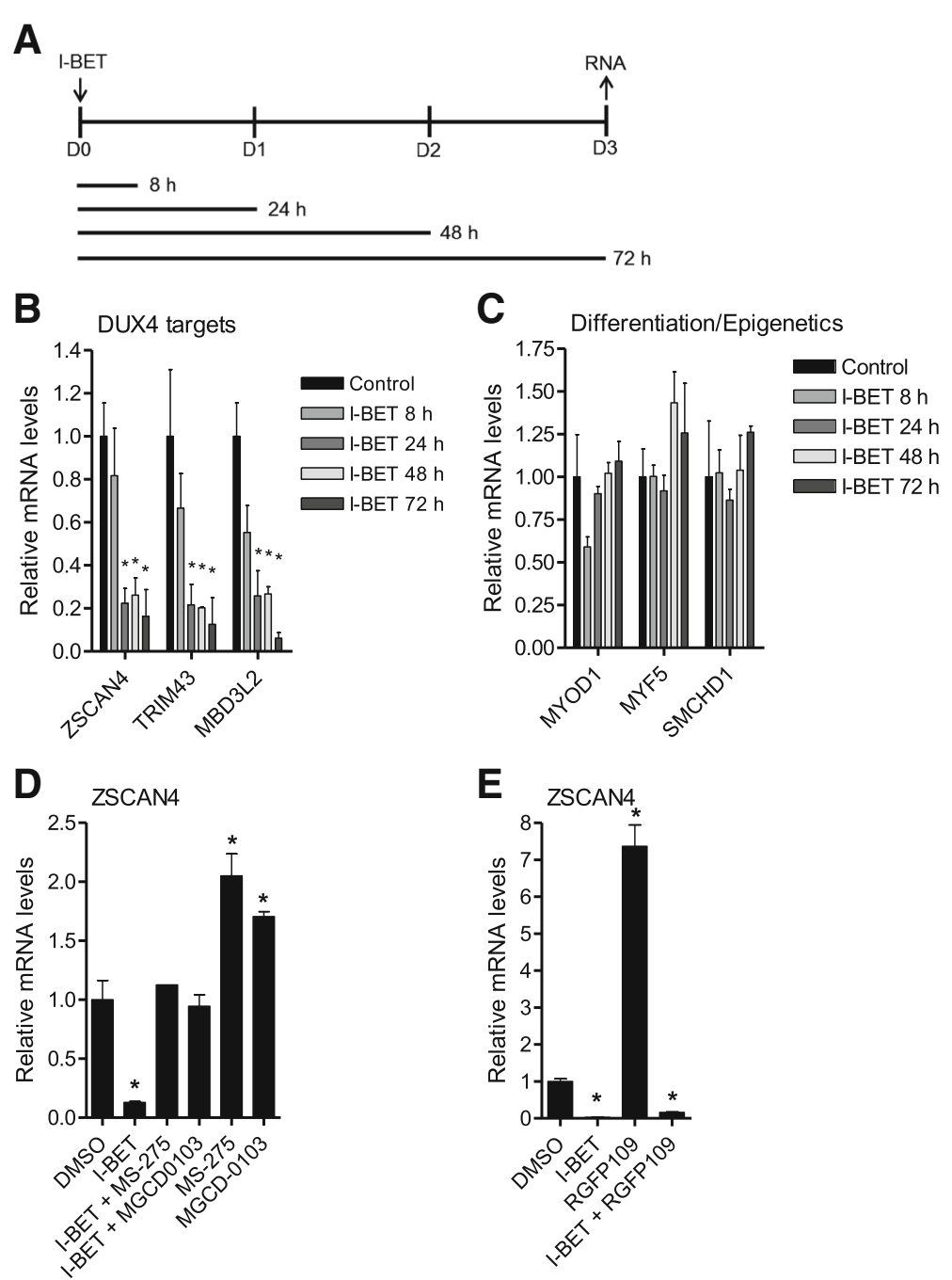

Fig. 2 BETi have a memory effect on DUX4 that is mediated by HDACs. a-c BETi cause sustained DUX4 repression. a Experimental timeline. Subconfluent MB200 FSHD2 myoblasts were treated with $1 \mu \mathrm{M}$ of the BETi I-BET762 (I-BET) on day 0 (D0) for 8, 24, 48, or $72 \mathrm{~h}$ and gene expression analyzed on day 3 (D3). $\mathbf{b}$ DUX4 target gene mRNA levels after treatment as in $\mathbf{a}$. $\mathbf{c}$ Expression of the myoblast lineage genes MYOD1 and MYF5 and the epigenetic modifier SMCHD1 after treatment as in $\mathbf{a}$. $\mathbf{d}$-e HDACi block the BETi-mediated memory effect. $\mathbf{d}$ Levels of the DUX4 target gene ZSCAN4 in MB200 FSHD2 myoblasts that were treated with the indicated compounds (DMSO control, $1 \mu$ M I-BET762, $2.5 \mu \mathrm{M}$ MS-275, $2.5 \mu \mathrm{M}$ MGCD0103) for $24 \mathrm{~h}$ and then cultured in fresh media for an additional $48 \mathrm{~h}$ before harvest. e ZSCAN4 expression after

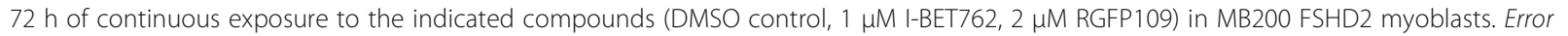
bars indicate the standard deviation from the mean of three biological replicates. $p$ values were calculated using a one-way analysis of variance with Dunnett's post test. ${ }^{*} p<0.05$

maintenance of chromosomes flexible hinge domain containing 1 (SMCHD1), the gene most commonly mutated in FSHD2 [11], were not affected (Fig. 2b-c). These data reveal a perdurance of BETi-mediated DUX4 repression lasting up to $48 \mathrm{~h}$ after drug removal, which we refer to as the BETi-mediated memory effect.

Class I histone deacetylases (HDACs) have been previously implicated in suppressing DUX4 expression [31]. Since BETi block binding of BET proteins to acetyl-lysine moieties $[29,30]$, we reasoned that the BETi-mediated memory effect on DUX4 expression might occur by a mechanism in which HDACs remove the exposed acetyl groups during BET protein displacement. Therefore, inhibition of the appropriate HDACs during the period of BETi exposure should block the memory effect of sustained DUX4 suppression. Indeed, co-incubation of I-BET762 with the class I HDAC inhibitors MS-275 or MGCD0103 [32] for 24 h, followed by $48 \mathrm{~h}$ without IBET762 or class I HDAC inhibitors, blocked the memory effect typically observed $48 \mathrm{~h}$ later (Fig. 2d). Additionally, a 24-h pulse of these class I HDAC inhibitors in the absence of I-BET762 stimulated DUX4 target gene expression at $72 \mathrm{~h}$ (Fig. 2d). These data are consistent with a dynamic role for class I HDACs in normally suppressing DUX4 
expression and in mediating the extended DUX4 repression by BETi that lasts at least $48 \mathrm{~h}$ after drug removal. Similarly, continuous exposure of MB200 FSHD2 myoblasts to the highly selective class I HDAC inhibitor RGFP109 [33] for $72 \mathrm{~h}$ resulted in an increase in DUX4 target expression (Fig. 2e). However, this induction was completely blocked when I-BET762 was included during the entire 72-h assay. These data suggest that increased expression of DUX4 during continuous inhibition of HDAC activity is associated with lysine acetylation and, since this increase is sensitive to BET inhibition, the recruitment of BET proteins for the activation of DUX4 transcription.

DUX4 de-repression in FSHD muscle coincides with a decrease in DNA methylation along the D4Z4 arrays [11, 34-36]. To determine if BETi-mediated suppression of DUX4 expression was associated with re-establishment of DNA methylation at the D4Z4 repeats, we propagated MB200 FSHD2 myoblasts for 3 weeks in the continuous presence of low dose (+)-JQ1. These conditions maintained a 95\% suppression of DUX4 targets that did not recover as long as compound was present (Additional file 7: Figure S5A). DNA methylation was measured along two regions of the D4Z4 repeat comprising 19 total CpG sites by bisulfite sequencing. Average methylation was significantly lower in FSHD2 cells than in control myoblasts, as expected, and continuous (+)-JQ1 exposure did not increase DNA methylation at the D4Z4 repeat in FSHD2 muscle (Additional file 7: Figure S5B-D). These data suggest that BETi block DUX4 expression independent of DNA methylation and that maintaining decreased DUX4 levels does not feedback to re-establish normal DNA methylation patterns at D4Z4 repeats.

Withdrawing (+)-JQ1 from MB200 FSHD2 cultures after 3 weeks of continuous treatment resulted in a slow recovery of DUX4 target gene expression, with ZSCAN4 mRNA reaching $20 \%$ of initial levels after $96 \mathrm{~h}$ of growth in drug-free media (Additional file 7: Figure S5E). This is consistent with earlier results (Fig. 2) and suggests a low rate of re-initiation of DUX4 expression after BETi withdrawal.

\section{BRD4 mediates the activity of BET bromodomain inhibitors on DUX4 expression}

The majority of disclosed BETi suppress the activity of all four mammalian BET proteins (BRD2, BRD3, BRD4, and bromodomain testis associated (BRDT)) [29]. We therefore examined the expression of BET genes in 546 control (non-FSHD) and 54-2 FSHD1 myoblasts and myotubes. Although BRD2, BRD3, and BRD4 were robustly expressed (cycle times of 21-25) in undifferentiated myoblasts, BRDT was almost undetectable (cycle times > 40) (Additional file 8: Figure S6A-C). BRD2, $B R D 3$, and $B R D 4$ were also mildly induced by differentiation in both control and FSHD1 myotubes, whereas BRDT was weakly induced only in FSHD1 myotubes, suggesting it is a DUX4 target gene. Our published chromatin immunoprecipitation-sequencing (ChIP-seq) and RNA-sequencing (RNA-seq) datasets generated from DUX4-expressing muscle cells [13, 37] suggest that the BRDT promoter is bound by DUX4, but show that very few full-length $B R D T$ transcripts are present (Additional file 8: Figure S6D). Together, these data indicate that only BRD2-4 are candidates for mediating DUX4 expression that is sensitive to BETi.

We next performed siRNA knockdown experiments in 54-2 FSHD1 myoblasts to determine the necessity of each BET family member in mediating $D U X 4$ expression. We confirmed that siRNAs targeting BRD2, BRD3, and BRD4 effectively depleted the relevant $\mathrm{mRNA}$ and protein (Fig. 3a-b). To assess the effect on DUX4 expression, DUX4 and three DUX4 target gene mRNAs were quantified by RT-qPCR. DUX4, ZSCAN4, MBD3L2, and TRIM43 were all selectively inhibited by BRD4 siRNAs, whereas BRD2 knockdown had inconsistent effects (decreasing DUX4 and TRIM43 levels while having no impact on ZSCAN4 or $M B D 3 L 2)$, and BRD3 depletion resulted in increases in all four genes (Fig. 3c). Overall, these results demonstrate that DUX4 expression (and thus expression of its downstream targets) is facilitated largely by BRD4 (and possibly by BRD2) in FSHD1 myoblasts, and indicate that these two BET proteins likely mediate the activity of BETi on DUX4.

\section{Second screen identifies beta- 2 adrenergic receptor agonists as repressors of DUX4 expression}

For the second screen of the larger Pharmakon 1600 library, we developed an outcome measure based on the expression of the endogenous DUX4 target gene MBD3L2 [13] using RT-qPCR, which introduced fewer variables into the screen than our initial transfected DUX4 reporter approach. Notably, the four hits from the epigenetic modifier library luciferase-based screen were all active in this second screen (Additional file 2: Figure S1). Although the second screen used an MB200 FSHD2 cell line and PCR detection of an endogenous DUX4 target gene rather than an FSHD1 line and luminescence measurement of a transfected DUX4 reporter, the major difference from the first screen was the library used. The Pharmakon library contains 1600 compounds that have reached clinical evaluation in the USA or internationally and includes advanced clinical compounds with known mechanisms of action. The compounds in this library are included in Additional file 9: Table S3. The library was initially screened at a concentration of $5 \mu \mathrm{M}$ (see Methods for a detailed description of assay execution and performance). Drugs that reduced $M B D 3 L 2$ expression by $>65 \%$ were considered preliminary candidates and further subjected to 5-point dilution 


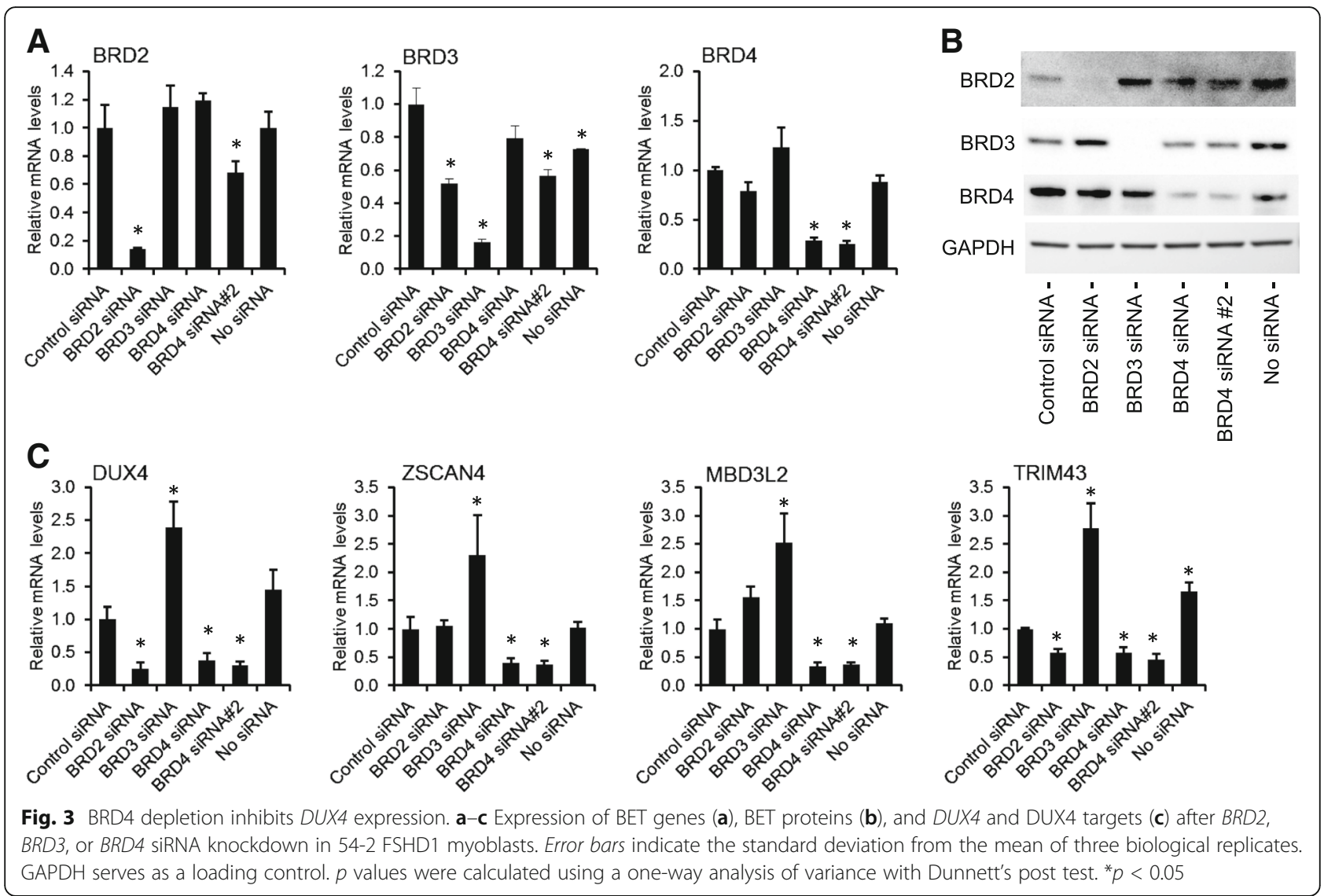

curves between 60 and $5000 \mathrm{nM}$ to determine $\mathrm{EC}_{50} \mathrm{~s}$. To eliminate molecules that reduced MBD3L2 expression due to toxicity or non-specific inhibition of muscle differentiation, cultures were scored on cytotoxicity and myotube formation by visual inspection, and $M B D 3 L 2$ levels were compared to those of the differentiation marker myosin heavy chain $(M Y H 2)$. Candidates were excluded when visual inspection indicated overt cytotoxicity (loss of adherent cells) and/or myotube formation was $<50 \%$ of normal.

This screening process resulted in 52 compounds that selectively reduced $M B D 3 L 2$ expression (Table 2). Adrenergic receptor agonists comprised $25 \%$ of these 52 compounds, and $46 \%$ of these specifically target the beta- 2 receptor (Table 2). These data indicate that adrenergic receptor activation, and in particular beta2 adrenergic receptor activation, results in decreased MBD3L2 expression during MB200 FSHD2 myotube differentiation. As a first validation step, selected adrenergic agonists were purchased as dry powders to ensure integrity and retested for their ability to modulate expression of MBD3L2 (Additional file 10: Table S4). These data confirmed the potent activity of many beta- 2 selective adrenergic agonists for decreasing $M B D 3 L 2$ in FSHD2 myotubes.
To further validate whether beta- 2 adrenergic agonists diminish MBD3L2 through inhibiting DUX4 expression, we analyzed mRNA for DUX4 and several DUX4 target genes in MB073 FSHD1 and MB200 FSHD2 cell lines. Formoterol treatment of proliferating myoblasts and differentiating myotubes reduced DUX4 expression and the expression of the DUX4 target genes ZSCAN4, TRIM43, and MBD3L2 (Fig. 4a-c). These effects occurred independent of any impact to myogenesis, which was monitored by visually inspecting cell cultures and measuring levels of the myogenic genes myogenin (MYOG) and creatine kinase, M-type $(C K M)$, without enhancing DUX4-mediated cell death (Additional file 11: Figure S7), and without affecting DUX4 transcriptional activity (Additional file 6: Figure S4). Beta-2 agonist-mediated reductions in DUX4 expression were evident within $1 \mathrm{~h}$ of adding formoterol to the culture media (Fig. 4d-e), though DUX4 repression was not sustained once the drug was removed (Fig. $4 \mathrm{f}-\mathrm{g}$ ).

The beta- 2 receptor mediates the activity of beta agonists on DUX4 expression

To determine the relative roles of the three beta receptors in mediating reductions in $D U X 4$ expression by beta 
Table 2 Screening hits from the Pharmakon 1600 library

\begin{tabular}{|c|c|c|c|}
\hline Drug & $\begin{array}{l}\text { MBD3L2 EC } 50 \\
\text { (nanomolar) }\end{array}$ & $\begin{array}{l}{\mathrm{MYH} 2 \mathrm{EC}_{50}} \\
\text { (nanomolar) }\end{array}$ & Mechanism \\
\hline Clenbuterol hydrochloride & $<60$ & $>5000$ & Beta-2 adrenergic agonist \\
\hline Tulobuterol hydrochloride & $<60$ & $>5000$ & Beta-2 adrenergic agonist \\
\hline Albuterol & $<60$ & $<60$ & Beta-2 adrenergic agonist \\
\hline Fenoterol hydrobromide & $<60$ & $<60^{\mathrm{e}}$ & Beta-2 adrenergic agonist \\
\hline Fluticasone & $<60$ & $<60^{d}$ & Glucocorticoid \\
\hline Nylidrin (Buphenine isoxsuprine) & $<60$ & $<60^{c}$ & Beta-2 adrenergic agonist \\
\hline Sirolimus & $<60$ & $<60$ & Immunosuppressant \\
\hline Terbutaline hemisulfate & $<60$ & $<60^{c}$ & Beta-2 adrenergic agonist \\
\hline Vinblastine & $<60^{b}$ & $<60$ & Antineoplastic, spindle poison \\
\hline Dequalinium & 70 & 5000 & Anti-infective, antineoplastic \\
\hline Bisoctrizole & $100^{c}$ & $>5000$ & Sunscreen \\
\hline Isoetharine & 100 & 5000 & Beta- 1 and beta- 2 adrenergic agonist \\
\hline Penicillamine & $180^{c}$ & $>5000$ & Chelating agent \\
\hline Benzethonium chloride & 190 & 400 & Anti-infective \\
\hline Cetylpyridinium chloride & 200 & 300 & Anti-infective \\
\hline Epinephrine bitartrate & 200 & 4000 & Alpha and beta-adrenergic agonist \\
\hline Phenylephrine & 200 & 4000 & Alpha-1 adrenergic agonist \\
\hline Piromidic acid & $<500^{\mathrm{a}}$ & $>5000$ & Antibacterial \\
\hline Acenocoumarol & $500^{b}$ & $>5000$ & Anticoagulant \\
\hline Atorvastatin & $500^{c}$ & $>5000$ & HmG-CoA reductase inhibitor \\
\hline Cresopirine & 500 & $>5000$ & Anti-inflammatory, fever reducer \\
\hline Dicoumarol & $500^{\mathrm{b}}$ & $>5000$ & Anticoagulant \\
\hline Thiostrepton & 500 & $700^{f}$ & Antibacterial \\
\hline Thimerosal & 500 & & Anti-infective, organomercury \\
\hline Ethylnorepinephrine & 600 & 5000 & Alpha and beta adrenergic agonist \\
\hline Fluvoxamine & 1000 & 4000 & SSRI, antidepressant \\
\hline Clozapine & 1000 & 2000 & Antipsychotic \\
\hline Adrenalone hydrochloride & 1000 & 1500 & Alpha-adrenergic agonist \\
\hline Butamben & $1000^{f}$ & 1000 & Local anesthetic \\
\hline Thonzonium & 1000 & 1000 & Mucolytic antibacterial \\
\hline Mebendazole & $1000^{f}$ & & Anthelmintic \\
\hline Puromycin & $1000^{f}$ & & Antibacterial, antineoplastic \\
\hline Colforsin & $1500^{d}$ & $>5000$ & Adenylyl cyclase activator \\
\hline Dimercaprol & 1500 & 3000 & Chelating agent \\
\hline Benzalkonium & 1500 & & Anti-infective \\
\hline Norepinephrine & 1600 & 4000 & Alpha and beta adrenergic agonist \\
\hline Polymyxin B & 1700 & 3500 & Antibacterial \\
\hline Oxedrine (synephrine) & 2000 & 5000 & $\begin{array}{l}\text { Alpha and beta adrenergic agonist } \\
\text { and antagonist }\end{array}$ \\
\hline Nonoxynol-9 & 2000 & & Spermicide \\
\hline Papaverine & 2500 & 10,000 & Vasodilator \\
\hline Pentamidine & 3000 & & Antiprotozoal \\
\hline Triamcinolone & 3000 & & Corticosteroid \\
\hline Dichlorephen & 3500 & & Anthelmintic \\
\hline
\end{tabular}


Table 2 Screening hits from the Pharmakon 1600 library (Continued)

\begin{tabular}{|c|c|c|c|}
\hline Ebselen & 4000 & & Antioxidant, anti-inflammatory \\
\hline Minocycline & 5000 & 10,000 & Antibacterial \\
\hline Inosine & 5000 & $>5000$ & Nucleoside \\
\hline Sulbentine & 5000 & $>5000$ & Antifungal \\
\hline Artesunate & 5000 & & Anti-malarial \\
\hline Broxaldine & 5000 & & Antifungal \\
\hline Broxyquinoline & 5000 & & Anti-infectant \\
\hline Meclocycline & 5000 & & Antibacterial \\
\hline Sennoside A & 5000 & & Cathartic/laxative \\
\hline
\end{tabular}

${ }^{a}$ Maximum $50 \%$ inhibition

${ }^{\mathrm{b}}$ Maximum $60 \%$ inhibition

${ }^{c}$ Maximum $65 \%$ inhibition

${ }^{\mathrm{d}}$ Maximum $70 \%$ inhibition

Maximum $85 \%$ inhibition

f Toxicity

agonists, we employed selective small molecule blockers of beta- 1 , beta- 2 , and beta- 3 adrenergic receptors. Neither the beta-1 selective antagonist atenolol [38] nor the beta-3 selective antagonist L-748,337 [39] affected the clenbuterol $\mathrm{EC}_{50}$ at relevant concentrations (Fig. 5a, c, d).
In contrast, the beta- 2 selective antagonist ICI-118,551 $[40,41]$ shifted the concentration response curves for clenbuterol rightward in a dose-dependent manner, resulting in a statistically significant increase in the apparent $\mathrm{EC}_{50}$ for clenbuterol (Fig. 5b, d).

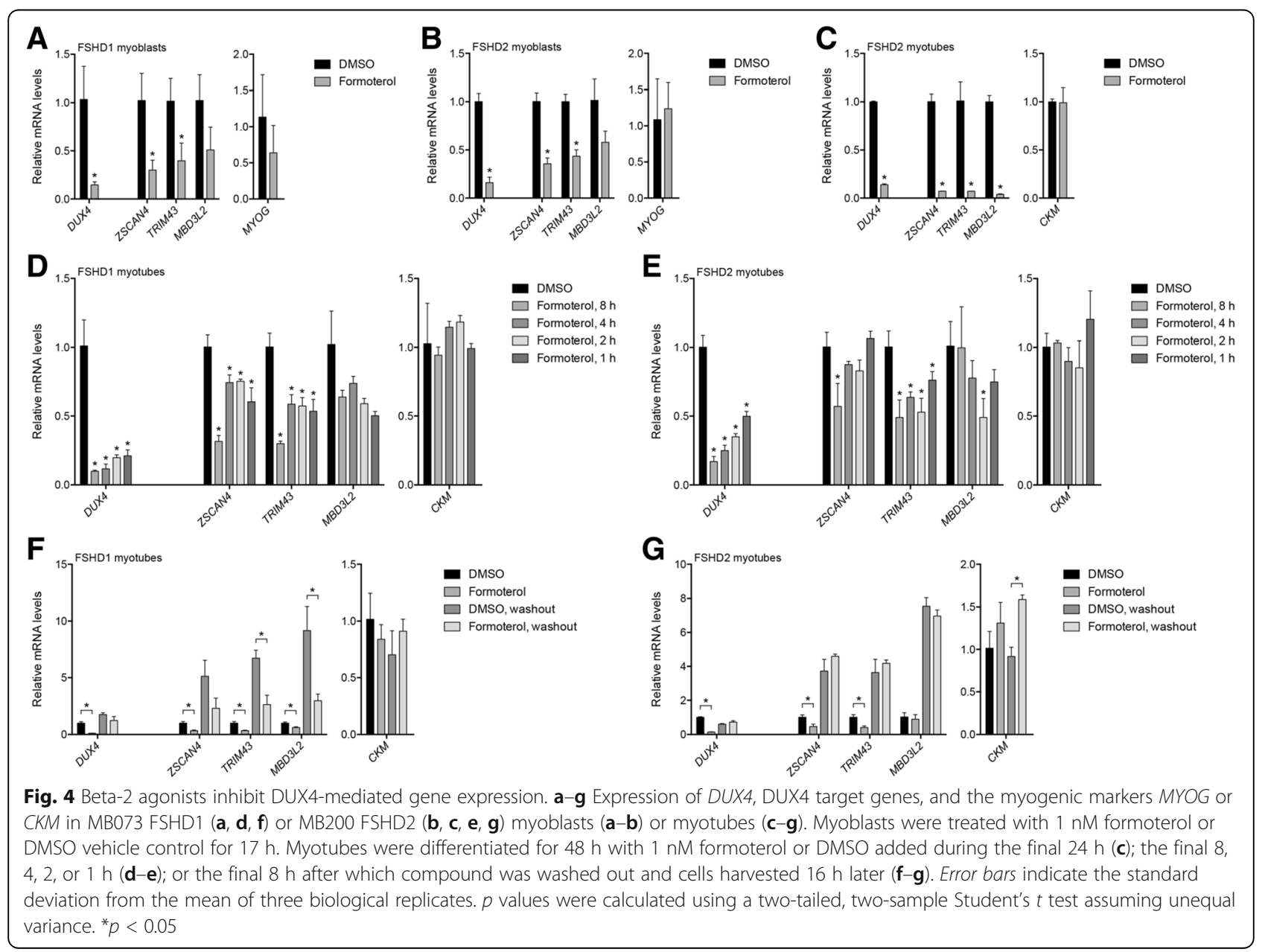




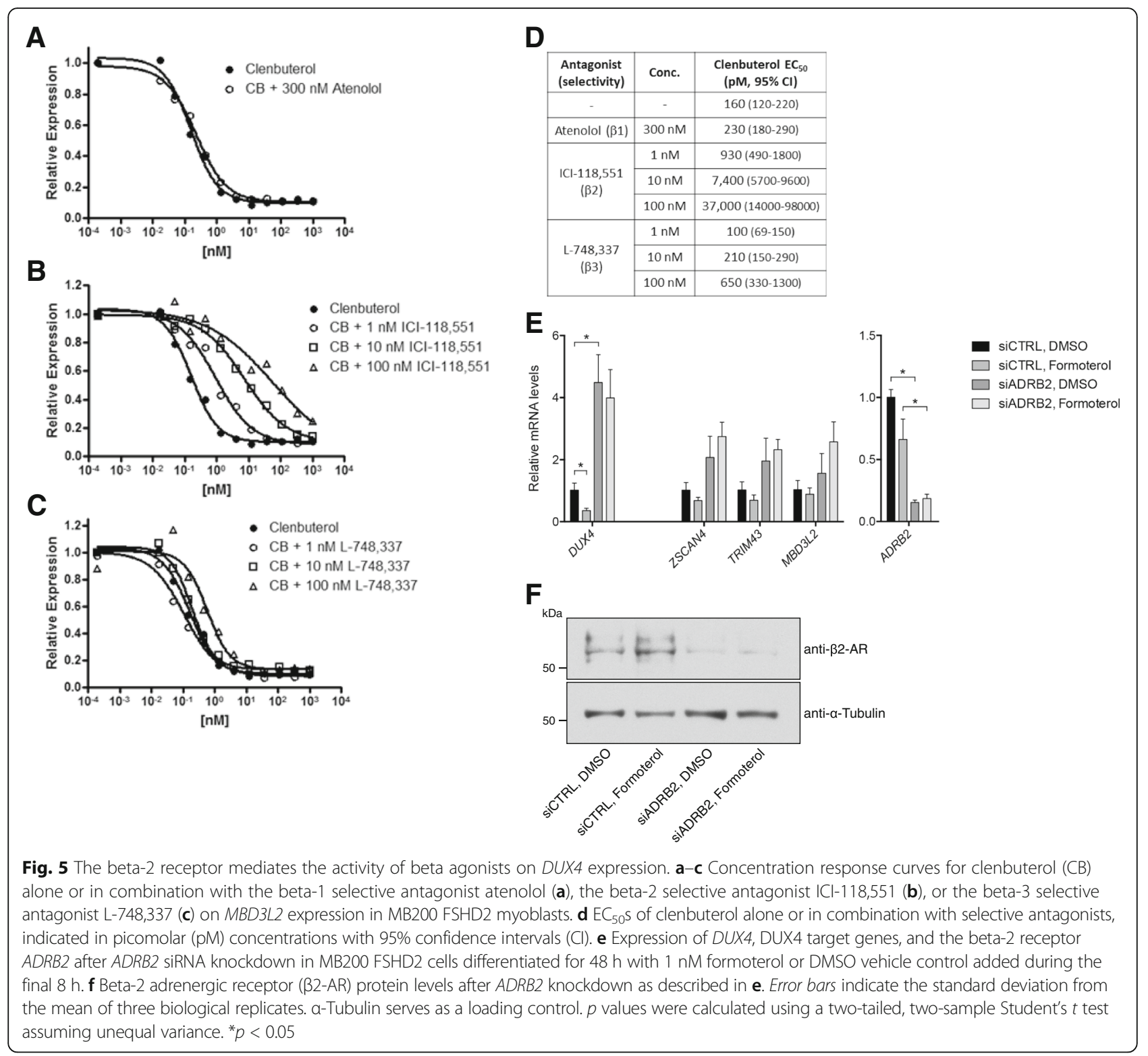

In addition, the conclusion that these compounds are acting as beta-2 agonists to inhibit DUX4 levels was further supported by several other observations: (1) a beta-3 selective adrenergic agonist (CGP-12177A) did not inhibit $M B D 3 L 2$; (2) a beta-2 agonist pro-drug (Bambuterol) was ineffective at blocking $M B D 3 L 2$ expression, consistent with its requirement to be metabolized into the fully active form for action on the beta- 2 receptor; and (3) phenylephrine, an alpha-1 selective adrenergic agonist, only weakly inhibited $M B D 3 L 2$, consistent with its reported weak off-target activity on the beta-2 receptor [42] (Additional file 10: Table S4). Together, these pharmacologic data suggest a major role for beta2 receptor activation in mediating the repressive effects on DUX4 expression.
Depleting the beta-2 adrenergic receptor by transfecting MB200 FSHD2 cells with siRNAs targeting the beta- 2 adrenergic receptor gene $A D R B 2$ genetically confirmed that beta agonists are reducing DUX4 expression via the beta- 2 adrenergic receptor (Fig. 5e-f). Upon siRNA knockdown of $A D R B 2$, the ability of formoterol to decrease $D U X 4$ expression was abolished. In addition, $A D R B 2$ depletion in the absence of any formoterol treatment resulted in an increase in DUX4 expression, suggesting that the beta- 2 adrenergic receptor is important for controlling DUX4 expression in the absence of synthetic ligands. Together with the pharmacologic data, these results demonstrate that beta-adrenergic agonist compounds act through the beta- 2 adrenergic receptor to decrease $D U X 4$ gene expression. 


\section{Pharmacologic modulation of the beta-2 receptor pathway}

Beta-2 agonist stimulation has long been known to promote muscle hypertrophy, in part by activating adenylyl cyclase to increase cellular cyclic adenosine monophosphate (cAMP) levels and stimulate protein kinase A (PKA) (for review see [43]). We used chemical modulators of these pathways to explore their effect on DUX4 expression. Forskolin, and its more water-soluble ana$\log \mathrm{NKH} 477$ (colforsin, a candidate inhibitor of DUX4 in screen 2, see Table 2), are activators of adenylyl cyclase. When MB200 FSHD2 myoblasts or myotubes were exposed to forskolin or NKH 477, DUX4 target gene expression was reduced (Fig. 6a-b). Additionally, the stable cAMP analog dibutyryl cAMP (dbcAMP) reduced DUX4 target expression in MB200 FSHD2 myoblasts and myotubes (Fig. $6 \mathrm{c}-\mathrm{d}$ ). These data indicate that cellular cAMP levels are important for regulating DUX4 expression and that beta-2 receptor agonists might suppress $D U X 4$ expression by modulating cAMP levels. In contrast, the PKA inhibitor $\mathrm{H}-89$ provided only marginal recovery from the inhibition of DUX4 mRNA by formoterol treatment in differentiating MB073 FSHD1 or MB200 FSHD2 myotubes, and H-89 exposure in the absence of formoterol did not increase DUX4 expression (Additional file 12: Figure S8). These results suggest that beta- 2 agonists may not primarily utilize the PKA pathway to achieve inhibition of DUX4 expression, and advance the possibility of PKAindependent regulation of the D4Z4 array.

\section{Discussion}

DUX4 mis-expression in skeletal muscle is ultimately responsible for muscle degeneration in FSHD. Therefore, suppressing $D U X 4$ expression is a primary therapeutic approach for halting FSHD disease progression, and identifying drug targets for this purpose is a critical step. Much FSHD research to date has focused on genetic changes and the resultant loss of epigenetic silencing of DUX4 within D4Z4 macrosatellite repeats in somatic tissue. However, a detailed mechanistic understanding of the process that results in transcriptional "bursts" of DUX4 in a subset of muscle cell nuclei $[5,18]$ is still lacking. Understanding this process is likely to produce drug targets with the potential to address the underlying cause of FSHD and the multiple mechanisms that contribute to disease pathology.

As a complementary approach to previous studies on genetic and epigenetic changes, we have used chemical genetics to identify signaling pathways and epigenetic machinery that directly or indirectly influence DUX4 expression in FSHD muscle. By screening libraries of chemical compounds with known mechanisms of action, we revealed unanticipated roles for BET proteins and beta- 2 adrenergic receptor signaling in the processes controlling DUX4 expression (Fig. 7). The novelty of these findings highlight the differences between the screens described here and those performed by others, who have used exogenous DUX4-mediated cytotoxicity in non-FSHD cells to identify broad inhibitors and mediators of cell death pathways [16, 22, 28], whereas our efforts uncovered
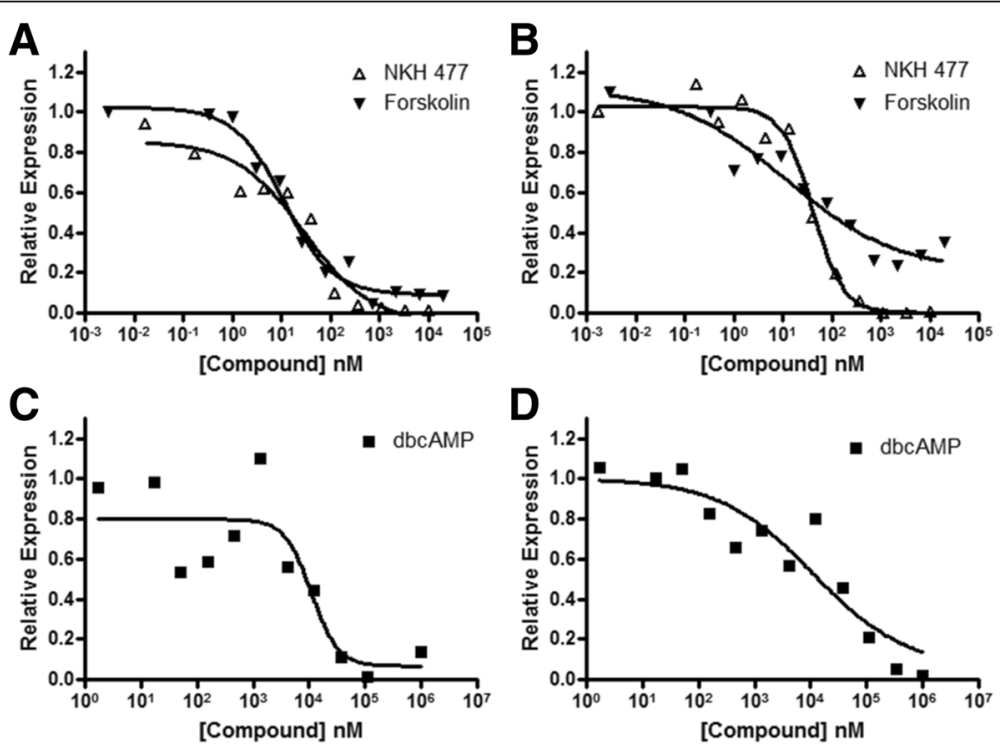

Fig. 6 Elevated cAMP levels inhibit DUX4 target gene expression. a-d Expression of the DUX4 target gene MBD3L2 in MB200 FSHD2 myoblasts $(\mathbf{a}, \mathbf{c})$ or myotubes $(\mathbf{b}, \mathbf{d})$ treated with the adenylyl cyclase activators NKH 477 or forskolin (a-b) or the cAMP analog dbcAMP (c-d) at the indicated concentrations. Data are normalized to untreated controls 


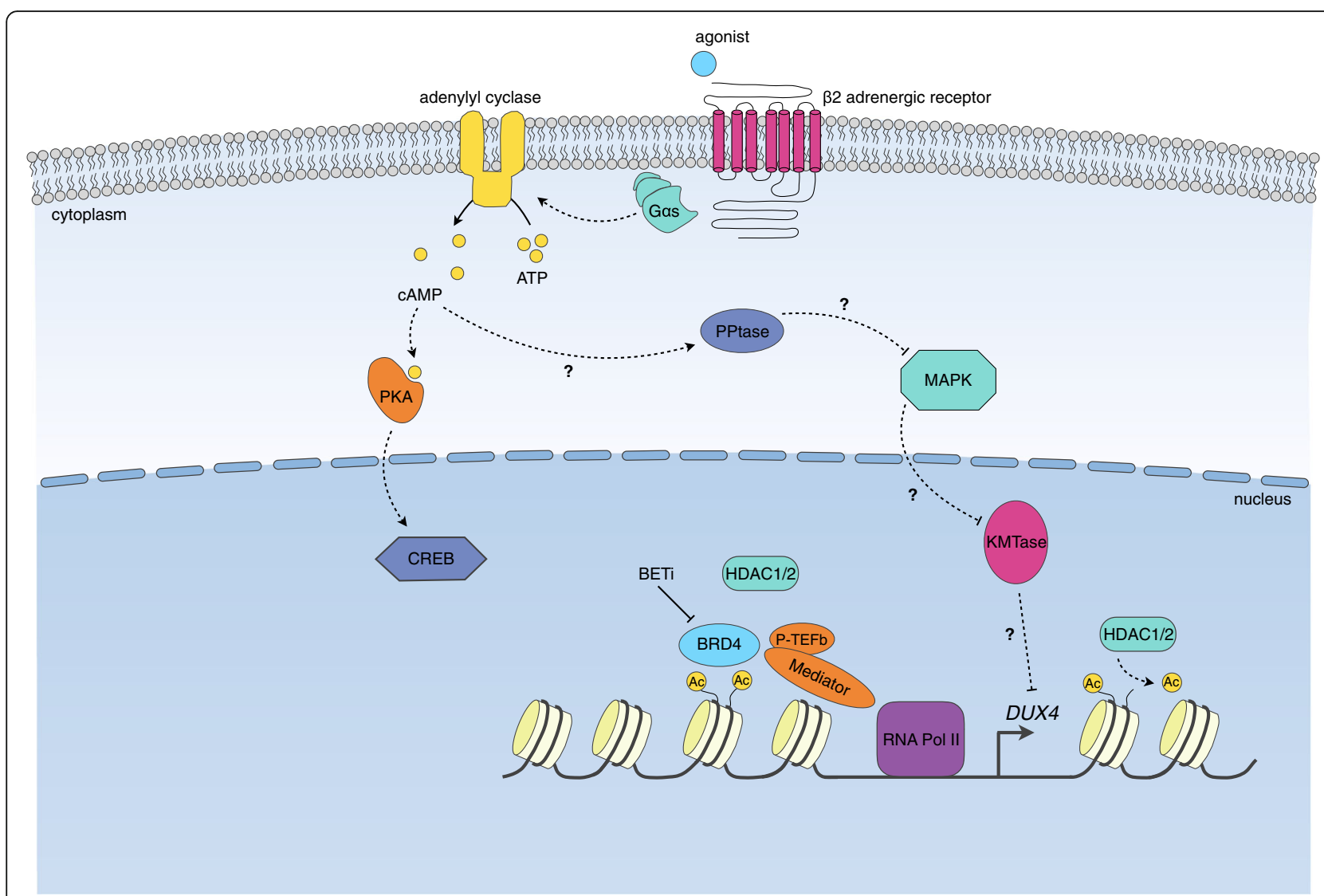

Fig. 7 Model of DUX4 regulation by BET proteins and beta-2 adrenergic signaling. BRD4 binds acetylated lysines at the D4Z4 array and recruits complexes such as P-TEFb and Mediator to facilitate transcriptional activation by RNA polymerase II. This is counteracted by the activity of HDAC1/2, which deacetylate lysines to inhibit gene induction. BRD4 binding to acetylated lysines may also shield these residues from HDAC1/2 activity. BETi prevent the binding of BRD4 to acetylated lysines and therefore block DUX4 expression while allowing HDAC1/2 access to the exposed acetyl groups. Beta-2 agonist binding to the beta-2 adrenergic receptor results in Gs G protein-mediated activation of adenylyl cyclase, which catalyzes the formation of CAMP. Downstream effectors of CAMP include PKA-dependent and PKA-independent pathways. The inhibitory effect of beta-2 agonists on DUX4 expression appears to be mostly mediated through PKA-independent pathways, possibly including the speculative one outlined here which is imagined to act through signaling molecules such as phosphatases (PPtases) and mitogen-activated protein kinases (MAPKs) to influence chromatin modifiers like lysine methyltransferases (KMTases) to impact transcription of the DUX4 gene

specific regulators of the D4Z4 array that potentially provide more precise ways to disrupt FSHD pathogenesis. Importantly, it is not clear that merely blocking the most downstream consequence of DUX4 expression-apoptosis-will prevent disease progression given the significant perturbations to fundamental cellular processes like RNA quality control, protein homeostasis, and the immune response that are present in DUX4-expressing muscle $[13,15,18,44]$. However, it is also important to note that because DUX4 expression has been reported in the testis, thymus, and possibly the skin $[5,6,45]$, even a targeted therapeutic approach which envisions utilizing a small molecule to inhibit DUX4 in FSHD muscle may also affect the normal function of some tissues, and potential side effects should be investigated.

Through our screens, we discovered that BETi prevent DUX4 expression in FSHD muscle cells, uncovering a role for the proteins BRD4 (and possibly BRD2) in regulating the D4Z4 array. This is perhaps not unexpected, given that BET proteins have been widely shown to facilitate gene activation by recruiting transcriptional regulatory complexes to acetylated chromatin [46]. Indeed, the D4Z4 repeat contains acetylated histone $\mathrm{H} 4$ nucleosomes that are bound by epigenetic "readers" and "erasers" of this modification including histone deacetylase 1 (HDAC1), histone deacetylase 2 (HDAC2), and BRD4 itself [31, 47, 48] (Campbell et al. under review) (Fig. 7). There is intense interest by pharmaceutical companies in the development of potent and selective BETi as therapeutics for a myriad of diseases, and current trials seek to attenuate BET activity in settings as diverse as oncology, diabetes, and atherosclerosis; indeed, several studies have already combined current standard of care with BETi [49]. Given this level of interest and effort, BETi are candidates for future FSHD clinical trials. While the safety profile of BETi in cancer and other indications has been good, it is 
perhaps too soon to predict whether or not BET inhibition would offer a sufficient benefit to side effect profile for a chronic indication like FSHD. Potential complications may arise due to the wide expression pattern of BET proteins and their centrality to many cellular functions, including insulin production, cytokine gene transcription, $\mathrm{T}$ cell differentiation, adipogenesis, and repression of latent viruses like HIV [50]. Therefore, choice of a BETi with properties tailored for FSHD will be critical going forward. These might include an optimized bromodomain binding profile for inhibiting DUX4 and appropriate pharmacokinetic properties for muscle exposure, among others.

Our screening approach also enabled us to uncover a role for beta- 2 adrenergic receptor signaling in modulating DUX4 expression. This finding is unexpected based on our current understanding of D4Z4 regulation, and creates an important opportunity for future mechanistic studies to define the beta- 2 adrenergic signaling pathways involved and uncover additional targets for drug development. Our experiments so far suggest that the canonical PKA signaling pathway downstream of the beta-2 receptor may not be mediating the effects on DUX4. Though restraint must be taken when interpreting these data as residual PKA activity may be enough to mediate DUX4 suppression over the time course of our assay, it opens up the possibility that beta- 2 agonists act on D4Z4 via alternative, PKA-independent pathways. It is interesting to speculate that these signaling cascades might impact chromatin modifiers that act on the D4Z4 array and therefore alter the epigenetic state of the locus (Fig. 7). Additionally, it is possible that alternative pathways will be more specific for D4Z4, and therefore have fewer potential side effects if they can be identified and targeted for therapeutic modulation. Interestingly, several clinical trials of the beta- 2 adrenergic agonist albuterol have already been carried out in FSHD patients based on the drug's known anabolic effects [51-54]. Although albuterol significantly increased muscle mass in these studies, this did not translate into functional improvements; however, better outcome measures to evaluate the effectiveness of such treatments for FSHD are needed before any potential therapy is disregarded. Such measures are being developed and tested, and include better FSHDrelevant functional measurements, self-reported measures of disease burden, and non-invasive imaging of disease progression [55-57].

Efforts to discover chemical inhibitors for use in the treatment of FSHD have lagged behind advances for other muscle disorders, likely due in part to the genetic complexity of this disease. However, the recent consensus of DUX4 as the causative factor in FSHD has provided a clear molecular target and allowed for the development of drug screens based on the activity of DUX4, such as measuring the expression of its target genes or reporters based on their promoters. Our screens used these two measures of DUX4 activity on different chemical libraries. The screens showed that endogenous activity of DUX4 in FSHD muscle cells can be used reliably to identify compounds that suppress DUX4 expression. The libraries represented distinct classes of molecules, and it is encouraging that both of these small libraries yielded compounds that modulated DUX4 expression and are already in clinical trials and/or use. This apparently high success rate provides hope that many additional candidate therapeutic compounds can be identified by a similar approach on larger and more complex libraries. The prospect of multiple initial therapeutic candidates makes it all the more imperative to not only actively prepare for clinical trials in FSHD, but to also anticipate that trials designed to efficiently compare compound efficacy without a full-scale clinical outcomes analysis will be important to prioritize which drugs to move to full clinical studies. As one example, multiple BETi compounds have been developed but it will take significant pre-clinical and focused clinical studies to determine which one(s), if any, to move forward into large-scale clinical trials, or how to assess BETi relative to other candidates, such as beta- 2 adrenergic agonists. After decades of uncertainty regarding the pathophysiology of FSHD, these are refreshing new challenges.

\section{Conclusions}

In summary, we identified small molecules that inhibit DUX4 by screening several chemical libraries in FSHD patient-derived muscle cells while interrogating DUX4 target gene expression. These molecules-BET bromodomain inhibitors and beta- 2 agonists-revealed an unexpected role for BET proteins and beta- 2 adrenergic receptor signaling in the regulation of the D4Z4 array in somatic cells, and provide promising initial candidate classes of compounds for FSHD therapeutic development.

\section{Additional files}

\section{Additional file 1: Table S1. Characteristics of cell lines used in this} study. (XLSX $31 \mathrm{~kb}$ )

Additional file 2: Figure S1. Primary screening data for the Pharmakon 1600 library. Quantitative PCR detection of DUX4 target gene MBD3L2 is plotted for each library plate (88 compounds per plate). Data for each plate is normalized to no drug controls ( $n=8$ ), which were set to 1 , as described in Methods. Plate means are indicated with horizontal lines and positive control data from each plate (I-BET762, $n=8)$ are collectively plotted in the rightmost column (Pos Controls). (PDF $126 \mathrm{~kb}$ )

Additional file 3: Table S2. Collection of compounds that target epigenetic modifier proteins. (XLSX $33 \mathrm{~kb}$ )

Additional file 4: Figure S2. The DUX4-responsive ZSCAN4 reporter system used for screening epigenetic modifier compounds. (A) Schematic depicting the ZSCAN4 promoter luciferase reporter vector (top) that includes four tandem DUX4 binding sites (D4BS), and a control reporter in which three of the four D4BS have been mutated (bottom). (B) Activity 
of the ZSCAN4 reporter vectors in 54-2 FSHD1 and 54-6 control (non-FSHD) myoblasts and 6 day differentiated myotubes. Error bars indicate the standard deviation from the mean of three biological replicates. (PDF $66 \mathrm{~kb}$ )

Additional file 5: Figure S3. BETi block DUX4 target gene expression in FSHD myoblasts. (A) The expression level of ZSCAN4 mRNA after $72 \mathrm{~h}$ of treatment with the BETi (+)-JQ1, I-BET762, or I-BET151 in 54-2 FSHD1 myoblasts. (B) The BETi RVX-208 inhibits ZSCAN4 and TRIM43 expression in FSHD2 MB200 myoblasts treated for $72 \mathrm{~h}$ with $\mathrm{EC}_{50} \mathrm{~S}$ of $350 \mathrm{nM}$ and $280 \mathrm{nM}$, respectively. Error bars indicate the standard deviation from the mean of three biological replicates. (PDF $53 \mathrm{~kb}$ )

Additional file 6: Figure S4. BETi and beta-2 adrenergic agonists do not suppress the transcriptional activation function of DUX4. 54-6 control (non-FSHD) myoblasts that lack DUX4 were transfected with a DUX4 expression vector and DUX4 activity monitored by measuring mRNA levels of the endogenous DUX4 target gene MBD3L2. Compounds were added $5 \mathrm{~h}$ after transfection, a time at which there is little detectable MBD3L2 expression, and mRNA levels assessed at $24 \mathrm{~h}$ after transfection. The level of MBD3L2 at $24 \mathrm{~h}$ is expressed as 'Fold Activation' over the level present at $5 \mathrm{~h}$, which was set to 1 . Compounds were added in a 12 point, 3 -fold dilution series to cover concentrations well above those required to inhibit DUX4 expression. The data point farthest to the left for each of the compounds represents a DMSO (no drug) control. The approximate $\mathrm{EC}_{50} \mathrm{~S}$ of the beta-2 adrenergic agonist clenbuterol $(\mathrm{X})$ and the BETi I-BET762 (Y) for blocking DUX4 expression in FSHD myoblasts are indicated on the $x$-axis. (PDF $31 \mathrm{~kb}$ )

Additional file 7: Figure S5. FSHD myoblasts grown continuously in low dose BETi maintain suppressed DUX4 target gene expression but do not re-establish D4Z4 repeat DNA methylation. (A) DUX4 target gene expression in MB200 FSHD2 myoblasts grown for three weeks in media containing DMSO or $100 \mathrm{nM}(+)-\mathrm{JQ} 1$ compared to gene expression in 54-6 control (non-FSHD) myoblasts. (B) Structure of the D4Z4 repeat unit showing regions analyzed by bisulfate sequencing in this study (ADS3747 and ADS1454) relative to the DUX4 open reading frame (ORF). ADS3747 spans positions 665-708 and ADS1454 spans positions 2230-2361 with respect to the start of the Kpnl site. The locations of previously published methylation-sensitive restriction sites and regions analyzed by bisulfite sequencing (DR1, DR2 and DR3) are indicated. (C-D) Average percent methylation across 9 CpG sites within ADS3747 (C) or 10 CpG sites within ADS1454 (D) in 54-6 control (non-FSHD, Normal), 54-2 FSHD1, and MB200 FSHD2 myoblasts, as well as MB200 FSHD2 myoblasts grown for three weeks in media containing DMSO or $100 \mathrm{nM}(+)-\mathrm{JQ} 1$. (E) Slow recovery of DUX4 target gene expression after BETi withdrawal. MB200 FSHD2 myoblasts grown for 3 weeks in $100 \mathrm{nM}(+)-J \mathrm{Q} 1$ were split and seeded onto culture plates in the absence of drug at $\sim 10 \%$ confluence to allow for continued growth. ZSCAN4 mRNA levels in untreated MB200 FSHD2 myoblasts (Control), MB200 FSHD2 myoblasts maintained continuously in drug (JQ1) and MB200 FSHD2 myoblasts grown for the indicated times after compound withdrawal are shown. Error bars in (A) and (E) indicate the standard deviation from the mean of three biological replicates. (PDF $122 \mathrm{~kb}$ )

Additional file 8: Figure S6. Characterization of BET gene expression. (A-C) 54-2 FSHD1 and 54-6 control (non-FSHD, Normal) myoblasts were induced to differentiate into myotubes and gene expression measured. Differentiation efficiency was determined by examining the early differentiation marker MYOG and the late differentiation marker MYH2 (A) As expected, DUX4 targets were strongly induced upon differentiation of FSHD1 but not control myoblasts (B). The levels of BET family member (BRD2, BRD3, BRD4, BRDT) mRNAs are shown in (C). (D) Tracks showing ChIP-seq and RNA-seq reads mapped to the BRDT locus in DUX4-expressing muscle cells. Error bars indicate the standard deviation from the mean of three biological replicates. (PDF $117 \mathrm{~kb}$ )

Additional file 9: Table S3. Compounds present in the Pharmakon 1600 library. (XLSX $125 \mathrm{~kb}$ )

Additional file 10: Table S4. Retesting of adrenergic receptor agonists. (XLSX $38 \mathrm{~kb}$ )

Additional file 11: Figure S7. The effect of formoterol on DUX4mediated cell death. MB135 control (non-FSHD) myoblasts that stably express a doxycycline-inducible DUX4 transgene [37] were used to test the effect of the beta-2 agonist formoterol on DUX4-mediated cell death. (A) Bright field images showing cell morphology after DUX4 expression at $24 \mathrm{~h}$ post doxycycline induction, with DMSO or $1 \mathrm{nM}$ formoterol added during the last $16 \mathrm{~h}$. (B) Cell counts from wells imaged in (A). (C) Expression of the DUX4 transgene and endogenous DUX4 target gene MBD3L2 from cells treated as in (A). (D) Western blot showing expression of exogenous DUX4, endogenous MBD3L2, and endogenous Histone H3 as a loading control from cells treated as in (A). Error bars indicate the standard deviation from the mean of three biological replicates. (PDF $1068 \mathrm{~kb})$

Additional file 12: Figure S8. PKA inhibition does not prevent formoterol-mediated inhibition of DUX4 expression. (A-B) Expression of DUX4, DUX4 target genes, and the myogenic marker CKM in MB073 FSHD1 (A) or MB200 FSHD2 (B) myotubes differentiated for $48 \mathrm{~h}$ and treated with $1 \mathrm{nM}$ formoterol, $10 \mathrm{uM} \mathrm{H89}$, or both compounds during the final $8 \mathrm{~h}$ of culturing. Error bars indicate the standard deviation from the mean of three biological replicates. $P$-values were calculated using a two-tailed, two-sample Student's t-test assuming unequal variance. *, $p<0.05$. (PDF $163 \mathrm{~kb})$

\section{Abbreviations}

ADRB2: Adrenoceptor beta 2; BET: Bromodomain and extra-terminal; BETi: BET bromodomain inhibitor; BRD2: Bromodomain-containing protein 2; BRD3: Bromodomain-containing protein 3; BRD4: Bromodomain-containing protein 4; BRDT: Bromodomain testis associated; CAMP: Cyclic adenosine monophosphate; CDK4: Cyclin-dependent kinase 4; ChIP-seq: Chromatin immunoprecipitation-sequencing; CKM: Creatine kinase, M-type; dbcAMP: Dibutyryl cAMP; DMEM: Dulbecco's Modified Eagle Medium; DMSO: Dimethyl sulfoxide; DUX4: Double homeobox 4; $\mathrm{EC}_{50}$ : Half maximal effective concentration; FSHD: Facioscapulohumeral dystrophy; HDAC: Histone deacetylase; HDAC1: Histone deacetylase 1; HDAC2: Histone deacetylase 2; hTERT: Human telomerase reverse transcriptase; MBD3L2: Methyl-CpG binding domain protein 3 like 2; mRNA: Messenger RNA; MYF5: Myogenic factor 5; MYH2: Myosin heavy chain; MYOD1: Myogenic differentiation 1; MYOG: Myogenin; PCR: Polymerase chain reaction; PKA: Protein kinase $A$; RNA-seq: RNA-sequencing; RPL13A: Ribosomal protein L13a; RPL27: Ribosomal protein L27; RPL30: Ribosomal protein L30; RT-qPCR: Quantitative reverse transcription $P C R$; siRNA: Small interfering RNA; SMCHD1: Structural maintenance of chromosomes flexible hinge domain containing 1; TRIM43: Tripartite motif containing 43; ZSCAN4: Zinc finger and SCAN domain-containing 4

\section{Acknowledgements}

We thank Mining Gu, Marshall Huang, Joseph Saad, Cynthia Francis, and Neal Modi for technical assistance and screening of compounds. We thank Liying Yan and Ann Meyer of EpigenDx for their pyrosequencing technical assistance and expertise. We thank the Structural Genomics Consortium for supplying chemical probes and Oleg Federov and Panagis Filippakopoulos for advice.

\section{Funding}

This work was funded by NIH/NIAMS grant R01AR045203 (SJT); NIH/NINDS grant P01NS069539 (RT, SMvdM, SJT, FMS); NIH training grants T32CA009657 (AEC), T32GM007270 (SCS), and T32HG00035 (SCS); Friends of FSH Research (SJT); FSH Society (YH, FMS); FSHD Canada Foundation (YH); Prinses Beatrix Spierfonds grant W.OP14-01 (SMvdM); and Spieren voor Spieren (SMvdM). The funders had no role in the study design; collection, analysis, and interpretation of data; decision to publish; or preparation of the manuscript.

\section{Availability of data and materials}

All data generated or analyzed during this study are included in this published article and its supplementary information files.

\section{Authors' contributions}

Conceptualization: AEC, SMvdM, SJT, and FMS. Data curation: AEC and FMS. Formal analysis: AEC, JO, and FMS. Funding acquisition: AEC, SCS, YH, RT, SMvdM, SJT, and FMS. Investigation: AEC, JO, MPY, JWZ, SCS, LS, NS, ST, YH, and FMS. Methodology: AEC, SJT, and FMS. Project administration: AEC, SJT, and FMS. Resources: RT and SMvdM. Supervision: AEC, SJT, and FMS. 
Validation: AEC, JO, MPY, JWZ, YH, and FMS. Visualization: AEC and FMS. Writing —original draft: AEC, SJT, and FMS. Writing - review and editing: AEC, JO, MPY, JWZ, SCS, LS, NS, ST, YH, RT, SMvdM, SJT, and FMS. All authors read and approved the final manuscript.

\section{Ethics approval and consent to participate}

This study used pre-existing de-identified human cell lines from approved repositories and was determined not to be Human Subjects Research by the Fred Hutchinson Cancer Research Center Institutional Review Board and the Saint Louis University Institutional Biosafety Committee. Muscle samples were obtained from the Fields Center at the University of Rochester (https:// www.urmc.rochester.edu/neurology/fields-center.aspx), and subjects gave informed written consent prior to sample use.

\section{Consent for publication}

Not applicable.

\section{Competing interests}

SIT and FMS have filed a provisional patent application titled "Inhibition of DUX4 expression using bromodomain and extra-terminal domain protein inhibitors," but otherwise the authors declare that they have no competing interests.

\section{Publisher's Note}

Springer Nature remains neutral with regard to jurisdictional claims in published maps and institutional affiliations.

\begin{abstract}
Author details
${ }^{1}$ Human Biology Division, Fred Hutchinson Cancer Research Center, Seattle, WA 98109, USA. ${ }^{2}$ Edward A. Doisy Department of Biochemistry and Molecular Biology, Saint Louis University, Saint Louis, MO 63104, USA. ${ }^{3}$ Molecular and Cellular Biology Program, University of Washington, Seattle, WA 98105, USA. ${ }^{4}$ Department of Neurology, University of Rochester Medical Center, Rochester, NY 14642, USA. ${ }^{5}$ Department of Human Genetics, Leiden University Medical Center, 2333 ZA Leiden, The Netherlands. ${ }^{6}$ Department of Neurology, University of Washington, Seattle, WA 98105, USA.
\end{abstract}

Received: 12 June 2017 Accepted: 14 August 2017

\section{Published online: 04 September 2017}

\section{References}

1. Tawil R, van der Maarel SM, Tapscott SJ. Facioscapulohumeral dystrophy: the path to consensus on pathophysiology. Skelet Muscle. 2014:4:12.

2. Hendrickson PG, Dorais JA, Grow EJ, Whiddon JL, Lim JW, Wike CL, Weaver BD, Pflueger C, Emery BR, Wilcox AL, et al. Conserved roles of mouse DUX and human DUX4 in activating cleavage-stage genes and MERVL/HERVL retrotransposons. Nat Genet. 2017:49:925-34

3. Whiddon JL, Langford AT, Wong CJ, Zhong JW, Tapscott SJ. Conservation and innovation in the DUX4-family gene network. Nat Genet. 2017:49:935-40.

4. De laco A, Planet E, Coluccio A, Verp S, Duc J, Trono D. DUX-family transcription factors regulate zygotic genome activation in placental mammals. Nat Genet. 2017:49:941-5.

5. Snider L, Geng LN, Lemmers RJ, Kyba M, Ware CB, Nelson AM, Tawil R, Filippova GN, van der Maarel SM, Tapscott SJ, Miller DG. Facioscapulohumeral dystrophy: incomplete suppression of a retrotransposed gene. PLoS Genet. 2010;6:e1001181.

6. Das S, Chadwick BP. Influence of repressive histone and DNA methylation upon D4Z4 transcription in non-myogenic cells. PLoS One. 2016;11:e0160022.

7. van Overveld PG, Lemmers RJ, Sandkuijl LA, Enthoven L, Winokur ST, Bakels F, Padberg GW, van Ommen GJ, Frants RR, van der Maarel SM. Hypomethylation of D4Z4 in 4q-linked and non-4q-linked facioscapulohumeral muscular dystrophy. Nat Genet. 2003;35:315-7.

8. Zeng W, de Greef JC, Chen YY, Chien R, Kong X, Gregson HC, Winokur ST, Pyle A, Robertson KD, Schmiesing JA, et al. Specific loss of histone H3 lysine 9 trimethylation and HP1gamma/cohesin binding at D4Z4 repeats is associated with facioscapulohumeral dystrophy (FSHD). PLoS Genet. 2009;5:e1000559.

9. Daxinger L, Tapscott SJ, van der Maarel SM. Genetic and epigenetic contributors to FSHD. Curr Opin Genet Dev. 2015;33:56-61.

10. Lemmers RJ, van der Vliet PJ, Klooster R, Sacconi S, Camano P, Dauwerse JG, Snider L, Straasheijm KR, van Ommen GJ, Padberg GW, et al. A unifying genetic model for facioscapulohumeral muscular dystrophy. Science. 2010;329:1650-3.

11. Lemmers RJ, Tawil R, Petek LM, Balog J, Block GJ, Santen GW, Amell AM, van der Vliet PJ, Almomani R, Straasheijm KR, et al. Digenic inheritance of an SMCHD1 mutation and an FSHD-permissive D4Z4 allele causes facioscapulohumeral muscular dystrophy type 2. Nat Genet. 2012:44:1370-4.

12. van den Boogaard ML, Lemmers RJ, Balog J, Wohlgemuth M, Auranen M, Mitsuhashi S, van der Vliet PJ, Straasheijm KR, van den Akker RF, Kriek M, et al. Mutations in DNMT3B modify epigenetic repression of the D4Z4 repeat and the penetrance of facioscapulohumeral dystrophy. Am J Hum Genet. 2016;98:1020-9.

13. Geng LN, Yao Z, Snider L, Fong AP, Cech JN, Young JM, van der Maarel SM, Ruzzo WL, Gentleman RC, Tawil R, Tapscott SJ. DUX4 activates germline genes, retroelements, and immune mediators: implications for facioscapulohumeral dystrophy. Dev Cell. 2012;22:38-51.

14. Young JM, Whiddon JL, Yao Z, Kasinathan B, Snider L, Geng LN, Balog J, Tawil R, van der Maarel SM, Tapscott SJ. DUX4 binding to retroelements creates promoters that are active in FSHD muscle and testis. PLoS Genet. 2013;9:e1003947.

15. Feng Q, Snider L, Jagannathan S, Tawil R, van der Maarel SM, Tapscott SJ, Bradley RK. A feedback loop between nonsense-mediated decay and the retrogene DUX4 in facioscapulohumeral muscular dystrophy. Elife. 2015:4:1-13.

16. Bosnakovski D, Xu Z, Gang EJ, Galindo CL, Liu M, Simsek T, Garner HR, AghaMohammadi S, Tassin A, Coppee F, et al. An isogenetic myoblast expression screen identifies DUX4-mediated FSHD-associated molecular pathologies. EMBO J. 2008;27:2766-79.

17. Kowaljow V, Marcowycz A, Ansseau E, Conde CB, Sauvage S, Matteotti C, Arias C, Corona ED, Nunez NG, Leo O, et al. The DUX4 gene at the FSHD1A locus encodes a pro-apoptotic protein. Neuromuscul Disord. 2007:17:611-23.

18. Rickard AM, Petek LM, Miller DG. Endogenous DUX4 expression in FSHD myotubes is sufficient to cause cell death and disrupts RNA splicing and cell migration pathways. Hum Mol Genet. 2015;24:5901-14

19. Wallace LM, Garwick SE, Mei W, Belayew A, Coppee F, Ladner KJ, Guttridge D, Yang J, Harper SQ. DUX4, a candidate gene for facioscapulohumeral muscular dystrophy, causes p53-dependent myopathy in vivo. Ann Neurol. 2011;69:540-52.

20. Winokur ST, Chen YW, Masny PS, Martin JH, Ehmsen JT, Tapscott SJ, van der Maarel SM, Hayashi Y, Flanigan KM. Expression profiling of FSHD muscle supports a defect in specific stages of myogenic differentiation. Hum Mol Genet. 2003;12:2895-907.

21. Snider L, Asawachaicharn A, Tyler AE, Geng LN, Petek LM, Maves L, Miller DG, Lemmers RJ, Winokur ST, Tawil R, et al. RNA transcripts, miRNA-sized fragments and proteins produced from D4Z4 units: new candidates for the pathophysiology of facioscapulohumeral dystrophy. Hum Mol Genet. 2009; 18:2414-30

22. Shadle SC, Zhong JW, Campbell AE, Conerly ML, Jagannathan S, Wong CJ, Morello TD, van der Maarel SM, Tapscott SJ. DUX4-induced dsRNA and MYC mRNA stabilization activate apoptotic pathways in human cell models of facioscapulohumeral dystrophy. PLoS Genet. 2017;13:e1006658.

23. Stadler G, Chen JC, Wagner K, Robin JD, Shay JW, Emerson CP Jr, Wright WE. Establishment of clonal myogenic cell lines from severely affected dystrophic muscles_CDK4 maintains the myogenic population. Skelet Muscle. 2011;1:12.

24. Livak KJ, Schmittgen TD. Analysis of relative gene expression data using real-time quantitative PCR and the 2(-Delta Delta $C(T))$ method. Methods. 2001;25:402-8.

25. Geng LN, Tyler AE, Tapscott SJ. Immunodetection of human double homeobox 4. Hybridoma (Larchmt). 2011;30:125-30.

26. Balog J, Thijssen PE, Shadle S, Straasheijm KR, van der Vliet PJ, Krom YD, van den Boogaard ML, de Jong A, Raj FL, Tawil R, et al. Increased DUX4 expression during muscle differentiation correlates with decreased SMCHD1 protein levels at D4Z4. Epigenetics. 2015;10:1133-42.

27. Yao Z, Snider L, Balog J, Lemmers RJ, Van Der Maarel SM, Tawil R, Tapscott SJ. DUX4-induced gene expression is the major molecular signature in FSHD skeletal muscle. Hum Mol Genet. 2014;23:5342-52.

28. Choi SH, Bosnakovski D, Strasser JM, Toso EA, Walters MA, Kyba M. Transcriptional inhibitors identified in a 160,000-compound small-molecule DUX4 viability screen. J Biomol Screen. 2016;21:680-8.

29. Filippakopoulos P, Qi J, Picaud S, Shen Y, Smith WB, Fedorov O, Morse EM, Keates T, Hickman TT, Felletar I, et al. Selective inhibition of BET bromodomains. Nature. 2010;468:1067-73. 
30. Picaud S, Da Costa D, Thanasopoulou A, Filippakopoulos P, Fish PV, Philpott M, Fedorov O, Brennan P, Bunnage ME, Owen DR, et al. PFI-1, a highly selective protein interaction inhibitor, targeting BET bromodomains. Cancer Res. 2013;73:3336-46.

31. Huichalaf C, Micheloni S, Ferri G, Caccia R, Gabellini D. DNA methylation analysis of the macrosatellite repeat associated with FSHD muscular dystrophy at single nucleotide level. PLoS One. 2014;9:e115278.

32. Buglio D, Younes A. Histone deacetylase inhibitors in Hodgkin lymphoma. Investig New Drugs. 2010;28(Suppl 1):S21-7.

33. Rai M, Soragni E, Chou CJ, Barnes G, Jones S, Rusche JR, Gottesfeld JM Pandolfo M. Two new pimelic diphenylamide HDAC inhibitors induce sustained frataxin upregulation in cells from Friedreich's ataxia patients and in a mouse model. PLoS One. 2010;5:e8825.

34. Hartweck LM, Anderson LJ, Lemmers RJ, Dandapat A, Toso EA, Dalton JC, Tawil R, Day JW, van der Maarel SM, Kyba M. A focal domain of extreme demethylation within D4Z4 in FSHD2. Neurology. 2013;80:392-9.

35. Jones Tl, King OD, Himeda CL, Homma S, Chen JC, Beermann ML, Yan C, Emerson CP Jr, Miller JB, Wagner KR, Jones PL. Individual epigenetic status of the pathogenic D4Z4 macrosatellite correlates with disease in facioscapulohumeral muscular dystrophy. Clin Epigenetics. 2015;7:37.

36. Jones TI, Yan C, Sapp PC, McKenna-Yasek D, Kang PB, Quinn C, Salameh JS, King OD, Jones PL. Identifying diagnostic DNA methylation profiles for facioscapulohumeral muscular dystrophy in blood and saliva using bisulfite sequencing. Clin Epigenetics. 2014;6:23.

37. Jagannathan S, Shadle SC, Resnick R, Snider L, Tawil RN, van der Maarel SM, Bradley RK, Tapscott SJ. Model systems of DUX4 expression recapitulate the transcriptional profile of FSHD cells. Hum Mol Genet. 2016;25:4419-31.

38. Tsuchihashi H, Nakashima $Y$, Kinami J, Nagatomo T. Characteristics of 125/-iodocyanopindolol binding to beta-adrenergic and serotonin-1B receptors of rat brain: selectivity of beta-adrenergic agents. Jpn J Pharmacol. 1990:52:195-200.

39. Candelore MR, Deng L, Tota L, Guan XM, Amend A, Liu Y, Newbold R, Cascieri MA, Weber AE. Potent and selective human beta(3)-adrenergic receptor antagonists. J Pharmacol Exp Ther. 1999;290:649-55.

40. Baker JG. The selectivity of beta-adrenoceptor antagonists at the human beta1, beta2 and beta3 adrenoceptors. Br J Pharmacol. 2005;144:317-22.

41. Bilski AJ, Halliday SE, Fitzgerald JD, Wale JL. The pharmacology of a beta 2selective adrenoceptor antagonist (ICI 118,551). J Cardiovasc Pharmacol. 1983:5:430-7.

42. Torp KD, Tschakovsky ME, Halliwill JR, Minson CT, Joyner MJ. Beta-receptor agonist activity of phenylephrine in the human forearm. J Appl Physiol (1985). 2001:90:1855-9.

43. Joassard OR, Durieux AC, Freyssenet DG. Beta2-adrenergic agonists and the treatment of skeletal muscle wasting disorders. Int J Biochem Cell Biol. 2013; 45:2309-21.

44. Homma S, Beermann ML, Boyce FM, Miller JB. Expression of FSHD-related DUX4-FL alters proteostasis and induces TDP-43 aggregation. Ann Clin Transl Neurol. 2015;2:151-66.

45. Dandapat A, Bosnakovski D, Hartweck LM, Arpke RW, Baltgalvis KA, Vang D, Baik J, Darabi R, Perlingeiro RC, Hamra FK, et al. Dominant lethal pathologies in male mice engineered to contain an X-linked DUX4 transgene. Cell Rep. 2014;8:1484-96.

46. Taniguchi Y. The bromodomain and extra-terminal domain (BET) family: functional anatomy of BET paralogous proteins. Int J Mol Sci. 2016;17:1-24.

47. Jiang G, Yang F, van Overveld PG, Vedanarayanan V, van der Maarel S, Ehrlich M. Testing the position-effect variegation hypothesis for facioscapulohumeral muscular dystrophy by analysis of histone modification and gene expression in subtelomeric 4q. Hum Mol Genet. 2003;12:2909-21.

48. Yang F, Shao C, Vedanarayanan V, Ehrlich M. Cytogenetic and immuno-FISH analysis of the $4 \mathrm{q}$ subtelomeric region, which is associated with facioscapulohumeral muscular dystrophy. Chromosoma. 2004;112:350-9.

49. Wang CY, Filippakopoulos P. Beating the odds: BETs in disease. Trends Biochem Sci. 2015:40:468-79.

50. Andrieu G, Belkina AC, Denis GV. Clinical trials for BET inhibitors run ahead of the science. Drug Discov Today Technol. 2016;19:45-50.
51. Kissel JT, McDermott MP, Mendell JR, King WM, Pandya S, Griggs RC, Tawil R. Randomized, double-blind, placebo-controlled trial of albuterol in facioscapulohumeral dystrophy. Neurology. 2001;57:1434-40.

52. Payan CA, Hogrel JY, Hammouda EH, Lacomblez L, Ollivier G, Doppler V, Eymard B, Attarian S, Pouget J, Desnuelle C, Laforet P. Periodic salbutamol in facioscapulohumeral muscular dystrophy: a randomized controlled trial. Arch Phys Med Rehabil. 2009;90:1094-101.

53. van der Kooi EL, Kalkman JS, Lindeman E, Hendriks JC, van Engelen BG, Bleijenberg G, Padberg GW. Effects of training and albuterol on pain and fatigue in facioscapulohumeral muscular dystrophy. J Neurol. 2007:254:931-40.

54. van der Kooi EL, Vogels OJ, van Asseldonk RJ, Lindeman E, Hendriks JC, Wohlgemuth M, van der Maarel SM, Padberg GW. Strength training and albuterol in facioscapulohumeral muscular dystrophy. Neurology. 2004:63:702-8.

55. Johnson NE, Quinn C, Eastwood E, Tawil R, Heatwole CR. Patient-identified disease burden in facioscapulohumeral muscular dystrophy. Muscle Nerve. 2012;46:951-3.

56. Statland JM, McDermott MP, Heatwole C, Martens WB, Pandya S, van der Kooi EL, Kissel JT, Wagner KR, Tawil R. Reevaluating measures of disease progression in facioscapulohumeral muscular dystrophy. Neuromuscul Disord. 2013:23:306-12.

57. Tawil R, Padberg GW, Shaw DW, van der Maarel SM, Tapscott SJ, Participants FW. Clinical trial preparedness in facioscapulohumeral muscular dystrophy: clinical, tissue, and imaging outcome measures 29-30 May 2015, Rochester. New York. Neuromuscul Disord. 2016;26:181-6.

\section{Submit your next manuscript to BioMed Central and we will help you at every step:}

- We accept pre-submission inquiries

- Our selector tool helps you to find the most relevant journal

- We provide round the clock customer support

- Convenient online submission

- Thorough peer review

- Inclusion in PubMed and all major indexing services

- Maximum visibility for your research

Submit your manuscript at www.biomedcentral.com/submit 